Pacific Journal of Mathematics

DIFFERENTIABILITY PROPERTIES OF SUBFUNCTIONS FOR
SECOND ORDER ORDINARY DIFFERENTIAL EQUATIONS 


\title{
DIFFERENTIABILITY PROPERTIES OF SUBFUNCTIONS FOR SECOND ORDER ORDINARY DIFFERENTIAL EQUATIONS
}

\author{
H. B. THOMPSON
}

\begin{abstract}
We obtain sharp differentiability results for subfunctions for second order ordinary differential equations $y^{\prime \prime}=f\left(x, y, y^{\prime}\right)$ on $[a, b]$. In the process we show that a subfunction satisfies a second order differential inequality similar to that satisfied by a lower solution. We show that a subfunction can be used in maximum principle arguments in the same way one uses a lower solution. As an application of these results we give necessary and sufficient conditions on a function in order that there is a differential equation for which it is a subfunction. We use our results together with the Perron method to improve on some existence results for two point boundary value problems obtained by Jackson, using Perron's method.
\end{abstract}

1. Introduction. Subfunctions and solutions of differential inequalities have been used for a long time to establish existence theorems and properties of solutions for both ordinary and partial differential equations. In 1915, Perron [11] used solutions of differential inequalities to establish the existence of a solution of the initial-value problem for the first order equation $y^{\prime}=f(x, y)$. In 1923 Perron [12] used subharmonic functions to study the Dirichlet problem for Laplace's equation for bounded plane domains. Perron used local solvability of the Dirichlet problem for circles and properties of subharmonic functions to prove the existence of a generalized solution which is harmonic in the interior of the domain, allowing the question of whether or not it assumes the specified values at the boundary to be treated separately. The success of subharmonic functions leads to various extensions of the concept and a careful study of the properties of these related functions. One early extension was to second order ordinary differential equations. We consider second order ordinary differential equations of the form

$$
y^{\prime \prime}=f\left(x, y, y^{\prime}\right)
$$

where $f:[a, b] \times \mathbf{R}^{2} \rightarrow \mathbf{R}$ is continuous.

By a solution of $(1.1)$ on a subinterval $I$ of $[a, b]$, we mean a function $y: I \rightarrow \mathbf{R}$ which is twice continuously differentiable on $I$ and satisfies 
(1.1) pointwise on $I$. We will frequently consider solutions $y$ of (1.1) defined on $I=\left[x_{1}, x_{2}\right]$ and satisfying the boundary conditions

$$
y\left(x_{1}\right)=y_{1}, \quad y\left(x_{2}\right)=y_{2} \text {. }
$$

In the context of solutions of (1.1) the analogue of a subharmonic function is a subfunction (see Jackson [9, Definition 3.1]).

A function $\alpha$ is said to be a subfunction for (1.1) on $[a, b]$ if for any $\left[x_{1}, x_{2}\right] \subset[a, b]$ all solutions $y$ of (1.1) and (1.2) satisfying

$$
y\left(x_{1}\right) \geq \alpha\left(x_{1}\right), \quad y\left(x_{2}\right) \geq \alpha\left(x_{2}\right)
$$

satisfy $y(x) \geq \alpha(x)$, for all $x \in\left[x_{1}, x_{2}\right]$. Superfunctions are defined by reversing these inequalities.

Thus a function $\alpha$ is a subfunction for $y^{\prime \prime}=0$ on $[a, b]$ iff $\alpha$ is convex.

The present work arose out of an attempt to improve on results of Jackson [9] concerning the applicability of the Perron method to establish existence of solutions for the boundary value problem (1.1) and

$$
y(a)=A, \quad y(b)=B .
$$

Some brief comments on Jackson's work are relevant. Following the Perron method, Jackson assumed there exists a subfunction $\alpha$ and a superfunction $\beta$ on $[a, b]$ such that

$$
\alpha(x) \leq \beta(x)
$$

on $[a, b]$, and

$$
\alpha(a) \leq A \leq \beta(a), \quad \alpha(b) \leq B \leq \beta(b) .
$$

He set

$$
\begin{aligned}
z(x)=\sup \{\Phi(x): \alpha(t) \leq & \Phi(t) \leq \beta(t), \text { for all } t \in[a, b] \\
\Phi(a) \leq & A, \Phi(b) \leq B, \\
& \Phi \text { a subfunction for }(1.1) \text { on }[a, b]\}
\end{aligned}
$$

and under additional assumptions on $f$ he showed $z$ was a solution. To show $z$ was a solution, Jackson first proved that a bounded subfunction has left and right handed limits at each point, as well as left and right handed derivatives (see [9, Theorems 4.1 and 4.3]). Assuming that all two point boundary value problems (1.1) and (1.2) have at most one solution, he then proved the following.

A function $w$ which is simultaneously a subfunction and a superfunction is a solution on an open subset $O$ of full measure and 
$\mathscr{D} \mathscr{L} w\left(x_{0}\right)=\mathscr{D} \mathscr{R} w\left(x_{0}\right)= \pm \infty$ for $x_{0}$ in the complement of $O$ (see [9, Theorem 4.10]). The function $z$ is simultaneously a subfunction and a superfunction ([9, Theorem 4.12]).

Under strong assumptions on $f$, he showed that there exist subfunctions and superfunctions satisfying (1.5) and (1.6), that two point boundary value problems have at most one solution and that $\mathscr{D} \mathscr{L} z\left(x_{0}\right)$ $=\mathscr{D R} z\left(x_{0}\right)= \pm \infty$ is impossible, so that $z$ is a solution on $(a, b)$ (see, [9, Theorem 4.17]). Then he constructed barriers at $a$ and $b$ when $A=B=0$, hence solving (1.1) and (1.4) in this case (see [9, Theorem 4.18]).

In this paper we improve on these results. In Theorems 3.6 and 3.7 we obtain sharp differentiability results for an arbitrary bounded subfunction and show that the uniqueness assumption is not necessary in [9, Theorem 4.10]. In Theorem 3.9 and Example 4.2 we show that if the uniqueness assumption in [9, Theorem 4.12] is weakened to a local uniqueness assumption, then $z$ need not be a superfunction but $z$ still has the smoothness properties [9, Theorem 4.10] guaranteed when $z$ is a superfunction. The local uniqueness property is satisfied by a wide class of equations. The local uniqueness property is satisfied by a wide class of equations and is often easier to verify. In Theorems 3.12 and 3.13 we use our results to improve on the existence theorems [9, Theorems 4.17 and 4.18]. A further advantage of the local uniqueness property is that our results apply in cases where there is more than one solution.

One of the most striking results of the paper is Theorem 3.2 showing that subfunctions satisfy the differential inequality (3.5) almost everywhere. A very important consequence of this and Theorem 3.6, illustrated in Lemma 3.5, is that subfunctions have all the properties required by a lower solution in order to use maximum principle arguments. We use this observation in Theorem 3.10 to give necessary and sufficient conditions on a function $\alpha$ in order that there is a differential equation for which it is a subfunction. It is also used to show our examples have their required properties.

We will use our results in a subsequent paper to prove further existence results and also to discuss the relationship between the various definitions of subfunctions and lower solution (see e.g. [1, 2]).

There is a literature concerned with showing that the properties of convex functions carry over to subfunctions for more general differential equations (see e.g. $[6,7,13,14]$ ). Most of the papers assume that all two point boundary value problems are uniquely solvable and show 
that some of the properties of convex functions carry over to subfunctions. In the special case that all two point boundary value problems are uniquely solvable, we show in Theorem 3.14 that a bounded subfunction has the same minimal smoothness properties as an arbitrary convex function.

2. Notation and preliminary results. Let $\Phi:[a, b] \rightarrow \mathbf{R}$. All limits below are taken in the extended reals. If $x \in[a, b)$ and $\lim _{t \rightarrow x^{+}} \Phi(t)$ exists, we denote it by $\Phi\left(x^{+}\right)$. Similarly if $x \in(a, b]$, we define $\Phi\left(x^{-}\right)$. Following Jackson [9] we make extensive use of the following modified left and right handed derivatives. If $x \in[a, b), \Phi\left(x^{+}\right)$exists in the reals, and moreover $\lim _{t \rightarrow x^{+}}\left(\Phi(t)-\Phi\left(x^{+}\right)\right) /(t-x)$ exists, then we denote it by $\mathscr{D} \mathscr{R} \Phi(x)$ and say $\Phi$ is right differentiable at $x$. Similarly we set $\mathscr{D} \mathscr{L} \Phi(x)=\lim _{t \rightarrow x^{-}}\left(\Phi(t)-\Phi\left(x^{-}\right)\right) /(t-x)$, where the appropriate limit exists. By $\mathscr{D} \mathscr{L} \Phi\left(x^{+}\right)$, we mean $\lim _{t \rightarrow x^{+}} \mathscr{D} \mathscr{L} \Phi(t)$ and similarly for other limits involving $\mathscr{D} \mathscr{L} \Phi(x)$ and $\mathscr{D} \mathscr{R} \Phi(x)$. As usual $D_{+} \Phi(x)$, $D^{+} \Phi(x), D_{-} \Phi(x)$ and $D^{-} \Phi(x)$ denote the Dini derivatives.

We set $\mathscr{D}^{ \pm} \Phi(x)=\lim \sup _{t \rightarrow x^{ \pm}}\left(\Phi(t)-\Phi\left(x^{ \pm}\right)\right) /(t-x)$ and $\mathscr{D}_{ \pm} \Phi(x)=$ $\liminf _{t \rightarrow x^{ \pm}}\left(\Phi(t)-\Phi\left(x^{ \pm}\right)\right) /(t-x)$ when these limits exist. If $\Phi$ is differentiable at $x$ in the extended reals, we denote the derivative by $\mathscr{D} \Phi(x)$ or $\Phi^{\prime}(x)$. For a set $S \subset \mathbf{R},|S|$ denotes its Lebesgue measure. Our other notation such as $C^{i}[a, b]$ is standard and needs no further comment.

To simplify the wording of statements and proofs of a number of results we adopt the convention that for a function $\Phi$ defined on $[a, b]$ $\Phi\left(a^{-}\right)=\Phi(a), \Phi\left(b^{+}\right)=\Phi(b)$ and $\mathscr{D} \mathscr{L} \Phi(a)=\mathscr{D} \mathscr{R} \Phi(a), \mathscr{D} \mathscr{R} \Phi(b)=$ $\mathscr{D} \mathscr{L} \Phi(b)$, when these latter derivatives exist.

For background results on boundary value problems, including some discussion of subfunctions, see [4].

The following well-known results are used frequently; their statements are included for clarity of presentation.

Theorem 2.1 ([9, Theorem 2.1]). Let $M>0$ and $N>0$ be given and let $q$ be the maximum of $\left|f\left(x, y, y^{\prime}\right)\right|$ on the compact set

$$
\left\{\left(x, y, y^{\prime}\right): a \leq x \leq b,|y| \leq 2 M,\left|y^{\prime}\right| \leq 2 N\right\} .
$$

If $\gamma=\operatorname{Min}\left[(8 M / q)^{1 / 2}, 2 N / q\right]$, then each boundary-value problem $y^{\prime \prime}=$ $f\left(x, y, y^{\prime}\right), y\left(x_{1}\right)=y_{1}, y\left(x_{2}\right)=y_{2}$ with $\left[x_{1}, x_{2}\right] \subset[a, b], 0<x_{2}-$ $x_{1} \leq \gamma,\left|y_{1}\right| \leq M,\left|y_{2}\right| \leq M,\left|\left(y_{2}-y_{1}\right) /\left(x_{2}-x_{1}\right)\right| \leq N$ has a solution $y(x) \in C^{2}\left[x_{1}, x_{2}\right]$. If $\varepsilon>0, \eta(M, N, \varepsilon)=\operatorname{Min}\left[(8 \varepsilon / q)^{1 / 2}, 2 \varepsilon / q, 1, \gamma\right] / 2$, 
$w(x)=y_{1}\left(x-x_{2}\right) /\left(x_{1}-x_{2}\right)+y_{2}\left(x-x_{1}\right) /\left(x_{2}-x_{1}\right)$ and $x_{2}-x_{1} \leq \eta$ then $|y(x)-w(x)|<\varepsilon$ and $\left|y^{\prime}(x)-w^{\prime}(x)\right|<\varepsilon$ on $\left[x_{1}, x_{2}\right]$.

See Jackson [9] for a proof.

THEOREM 2.2. Let $M>0$ and $N>0$ be given and let $Q$ be the maximum of $\left|f\left(x, y, y^{\prime}\right)\right|$ on the compact set $\left\{\left(x, y, y^{\prime}\right): a \leq x \leq b\right.$, $\left.|y| \leq M+1,\left|y^{\prime}\right| \leq N+1\right\}$. Let $c \in[a, b],\left|y_{0}\right| \leq M,\left|y_{0}^{\prime}\right| \leq N$ and $\varepsilon>0$ be given. If $\kappa(M, N, \varepsilon)=\min \{1, \varepsilon\}(N+Q+1)^{-1}$ there exists a solution $y$ of the initial-value problem $y^{\prime \prime}=f\left(x, y, y^{\prime}\right), y(c)=y_{0}$, $y^{\prime}(c)=y_{0}^{\prime}$ which is defined on $I_{\kappa}=[c-\kappa, c+\kappa] \cap[a, b]$ and satisfies

$$
\left|y(x)-y_{0}\right| \leq \varepsilon \text { and }\left|y^{\prime}(x)-y_{0}^{\prime}\right| \leq \varepsilon
$$

there. Furthermore every solution of this initial value problem is defined and satisfies (2.1) on $I_{\kappa}$.

The following lemmas will be used to simplify the proofs of the later results.

Corollary 2.3. Let $\alpha$ be a subfunction for $y^{\prime \prime}=f\left(x, y, y^{\prime}\right)$ on $[a, b]$. Let $M>0, N>0$, and $\varepsilon>0, a \leq x_{1}<x_{2} \leq b$ and $x_{2}-x_{1} \leq \eta(M, N, \varepsilon)$ where $\left|\alpha\left(x_{1}^{+}\right)\right|<M,\left|\alpha\left(x_{2}\right)\right| \leq M$ and $\left|\left(\alpha\left(x_{2}\right)-\alpha\left(x_{1}^{+}\right)\right) /\left(x_{2}-x_{1}\right)\right|$ $<N$. There is a solution $y$ on $\left[x_{1}, x_{2}\right]$ with $y\left(x_{1}\right)=\alpha\left(x_{1}^{+}\right)$and $y\left(x_{2}\right)=\alpha\left(x_{2}\right)$ satisfying $y(x) \geq \alpha(x)$, for all $x \in\left(x_{1}, x_{2}\right]$. Moreover $\left|y^{\prime}(x)-\left(\alpha\left(x_{2}\right)-\alpha\left(x_{1}^{+}\right)\right) /\left(x_{2}-x_{1}\right)\right| \leq \varepsilon$, for all $x \in\left[x_{1}, x_{2}\right]$.

Proof. Choose $n>0$ such that

$\frac{1}{n}<M-\left|\alpha\left(x_{1}^{+}\right)\right|$and $\frac{1}{n}\left(x_{2}-x_{1}\right)<N-\left|\left(\alpha\left(x_{2}\right)-\alpha\left(x_{1}^{+}\right)\right) /\left(x_{2}-x_{1}\right)\right|$;

then let $y_{i}$ be a solution on $\left[x_{1}, x_{2}\right]$ with $y_{i}\left(x_{1}\right)=\alpha\left(x_{1}^{+}\right)+1 /(n+i)$, $y_{i}\left(x_{2}\right)=\alpha\left(x_{2}\right)$, for $i=1,2, \ldots$. The existence of $y_{i}$ follows from Theorem 2.1. Moreover from the proof of that theorem it follows that a subsequence converges in $C^{1}\left[x_{1}, x_{2}\right]$ to a solution $y$ satisfying $y(x) \geq$ $\alpha(x)$ on $\left[x_{1}, x_{2}\right]$. By Theorem 2.1,y has the required properties.

As mentioned earlier subfunctions for $y^{\prime \prime}=0$ are convex functions. The next four results are needed to show that a subfunction restricted to a suitable open subset of full measure has the same minimal smoothness properties as an arbitrary convex function.

REMARK. Left handed and right handed limit results are interchangeable as are subfunction and superfunction results using suitable 
reflections. Moreover, using translation and rotation we need consider only results at $x=0$ and we may prescribe limits including $\mathscr{D} \mathscr{R} \alpha(0)$, for example, provided they exist and are finite.

The following result may be found in Jackson [9, Theorems 4.1 and 4.3, Corollary 4.2]. We include a proof for the sake of completeness and the convenience of the reader.

THEOREM 2.4. Let $\alpha$ be a bounded subfunction on $[a, b]$. If $a \leq c<b$ then $\alpha\left(c^{+}\right)$exists and $\mathscr{D} \mathscr{R} \alpha(c)$ exists in the extended reals. Moreover $\alpha(c) \leq \max \left\{\alpha\left(c^{-}\right), \alpha\left(c^{+}\right)\right\}$.

Proof. First we show that $\alpha\left(c^{+}\right)$exists. Assume that

$$
l=\lim _{x \rightarrow c^{+}} \inf \alpha(x)<\lim _{x \rightarrow c^{+}} \sup \alpha(x)=m .
$$

Then, by Theorem 2.2, choosing $\varepsilon>0, y_{0}^{\prime}=0$, and $y_{0} \in(l, m)$, there is $\kappa>0$ and a solution $y$ of the initial-value problem (1.1) with $y(c)=y_{0}$, and $y^{\prime}(c)=y_{0}^{\prime}$ on $I_{\kappa}$. Thus there exists $x_{1} \in(c, c+\kappa) \cap[a, b]$ such that $\alpha\left(x_{1}\right)<y\left(x_{1}\right), x_{2} \in\left(c, x_{1}\right)$ such that $\alpha\left(x_{2}\right)>y\left(x_{2}\right)$, and $x_{2} \in\left(c, x_{2}\right)$ such that $\alpha\left(x_{3}\right)<y\left(x_{3}\right)$. This contradicts the assumption that $\alpha$ is a subfunction on $[a, b]$ and thus $\alpha\left(c^{+}\right)$exists.

We show now that $\mathscr{D} \mathscr{R} \alpha(c)$ exists. Assume that

$$
l=\mathscr{D}_{+} \alpha(c)<\mathscr{D}^{+} \alpha(c)=m .
$$

By the above remark we may assume that $\alpha\left(c^{+}\right)=0$ and $l<-\varepsilon<\varepsilon<$ $m$ for some $\varepsilon>0$. Again by Theorem 2.2, choosing $y_{0}=0=y_{0}^{\prime}$, there is $\kappa>0$ and a solution of the initial value problem (1.1), $y(0)=0=$ $y^{\prime}(0)$ such that $\left|y^{\prime}(x)\right| \leq \varepsilon$ in $I_{\kappa}$. Thus there is $x_{1} \in(c, c+\kappa) \cap[a, b]$ such that $\alpha\left(x_{1}\right)<-\varepsilon x_{1} \leq y\left(x_{1}\right), x_{2} \in\left(c, x_{1}\right)$ such that $y\left(x_{2}\right) \leq \varepsilon x_{2}<\alpha\left(x_{2}\right)$, and $x_{3} \in\left(c, x_{2}\right)$ such that $\alpha\left(x_{3}\right)<-\varepsilon x_{3} \leq y\left(x_{3}\right)$. Again we have a contradiction, and the result follows.

The last part follows by an argument similar to the above and its proof is omitted.

THEOREM 2.5. Let $\alpha$ be a bounded subfunction for $y^{\prime \prime}=f\left(x, y, y^{\prime}\right)$ on $[a, b] . \quad$ If $\min \left\{\alpha(c), \alpha\left(c^{-}\right)\right\}<\alpha\left(c^{+}\right)$, then $\mathscr{D} \mathscr{R} \alpha(c)=\infty$. If $\min \left\{\alpha(c), \alpha\left(c^{+}\right)\right\}<\alpha\left(c^{-}\right)$, then $\mathscr{D} \mathscr{L} \alpha(c)=-\infty$. If $\mathscr{D} \mathscr{L} \alpha(c)=\infty$, then $\min \left\{\alpha(c), \alpha\left(c^{+}\right)\right\} \geq \alpha\left(c^{-}\right)$and $\mathscr{D} \mathscr{R} \alpha(c)=\infty$. If $\mathscr{D} \mathscr{R} \alpha(c)=-\infty$, then $\min \left\{\alpha(c), \alpha\left(c^{-}\right)\right\} \geq \alpha\left(c^{+}\right)$and $\mathscr{D} \mathscr{L} \alpha(c)=-\infty$.

Proof. Assume that $\min \left\{\alpha(c), \alpha\left(c^{-}\right)\right\}<\alpha\left(c^{+}\right)$and that $\mathscr{D} \mathscr{R} \alpha(c)<$ $\infty$. By the above remark we may assume that $c=0=\alpha\left(0^{+}\right)$ 
and $\mathscr{D R} \alpha(0)<-1$. Choose $\gamma \in(0, \min \{1, \eta(1,1,1)\})$ such that $\min \left\{\alpha\left(0^{-}\right), \alpha(0)\right\}<\alpha(\gamma)-\gamma$. By Corollary 2.3 the boundary value problem (1.1), $y(0)=\alpha(\gamma)=y(\gamma)$ has a solution $y$ satisfying $\alpha(x) \leq$ $y(x)$ on $(0, \gamma]$ and $\left|y^{\prime}(x)\right|<1$ on $[0, \gamma]$. Thus $0>y(0) \geq \alpha\left(0^{+}\right)$, a contradiction, and $\mathscr{D} \mathscr{R} \alpha(c)=\infty$.

As the other results follow by similar arguments, we omit their proofs.

In the special case $f\left(x, y, y^{\prime}\right)$ is such that all solutions of boundaryvalue problems, when they exist, are unique, the results of Theorem 2.5 may be found in Jackson [9] following the proof of his Theorem 4.10 .

THEOREM 2.6. If $\alpha$ is a bounded subfunction for $y^{\prime \prime}=f\left(x, y, y^{\prime}\right)$ on $[a, b]$ then $\mathscr{D R} \alpha(c) \geq \mathscr{D} \mathscr{L} \alpha(c)$, for all $c \in(a, b)$.

Proof. Assume that $\alpha$ is continuous at $c$ and $m=\mathscr{D} \mathscr{R} \alpha(c)<$ $\mathscr{D} \mathscr{L} \alpha(c)=l$. By the above remark we may assume that $c=0$, $\alpha(0)=0$ and $m<-\varepsilon<\varepsilon<l$ for some $\varepsilon \in(0,1)$. There exists $\kappa \in(0, \eta(1,1, \varepsilon) / 2)$ such that $\alpha( \pm \kappa)<-\varepsilon|\kappa|$. By Theorem 2.1 the boundary-value problem (1.1) with $y( \pm \kappa)=-\varepsilon|\kappa|$ has a solution $y$ satisfying $\left|y^{\prime}(x)\right|<\varepsilon$ on $[-\kappa, \kappa]$. Thus $y( \pm \kappa)>\alpha( \pm \kappa)$ while $y(0)<0=\alpha(0)$, a contradiction. Thus the result follows for $\alpha$ continuous at $c$.

If $\alpha$ is discontinuous at $c \in(a, b)$ the result follows from Theorem 2.5.

THEOREM 2.7. If $\alpha$ is a bounded subfunction for $y^{\prime \prime}=f\left(x, y, y^{\prime}\right)$ on $[a, b]$ then $\alpha^{\prime}$ exists in the extended reals except for a countable set of points $N \subset[a, b]$. Moreover, $\alpha^{\prime}$ is real valued almost everywhere in $(a, b)$.

Proof. From Theorem 2.4 it follows that $\alpha$ is continuous except at a countable set of points $M \subset[a, b]$. For $c \in\{(a, b) \backslash M\} \cap N$, $\mathscr{D} \mathscr{L} \alpha(c)<\mathscr{D} \mathscr{R} \alpha(c)$, thus there exists a rational number $q \in(\mathscr{D} \mathscr{L} \alpha(c)$, $\mathscr{D R} \alpha(c)$ ). Thus $\Phi(x)=\alpha(x)-q x$ satisfies $\mathscr{D} \mathscr{L} \Phi(c)<0<\mathscr{D} \mathscr{R} \Phi(c)$, so $\Phi$ has a strict local minimum at $c$. As a function can have at most a countable number of strict minima, it follows that $N$ is countable.

As noted in [9, Corollary 4.4] the second assertion follows from Theorem 2.4 and classical results of Denjoy-Young-Saks (see [15] p. 17 ) in the theory of functions of a real variable. 
REMARK. If $\alpha$ is an arbitrary subfunction for $y^{\prime \prime}=f\left(x, y, y^{\prime}\right)$ on $[a, b]$, then the conclusions of Theorems 2.4, 2.6 and 2.7 hold except that $\mathscr{D} \mathscr{R} \alpha(c)$ and $\mathscr{D} \mathscr{L} \alpha(c)$ are not defined if $\alpha\left(c^{+}\right)= \pm \infty$ and $\alpha\left(c^{-}\right)=$ $\pm \infty$, respectively.

To see this note that $\alpha\left(x^{+}\right)$and $\alpha\left(x^{-}\right)$exist in the extended reals by the proof of Theorem 2.4. Thus $\alpha$ has at most a countable number of discontinuities. The result follows from the Denjoy-Young-Saks theorem (see [15, p. 17]) and the proofs of Theorems 2.4, 2.5 and 2.7.

We will restrict our attention to bounded subfunctions; however the above remark can be used to extend a number of our results to the unbounded case.

REMARK. If $\alpha$ is a subfunction on $[a, b], c \in[a, b]$ and

$$
\Psi(x)= \begin{cases}\alpha(x) & \text { if } x \neq c \\ \alpha\left(c^{+}\right) & \text {if } x=c,\end{cases}
$$

then $\Psi$ need not be a subfunction as the following example shows.

EXAMPLE 2.8. Consider $y^{\prime \prime}=-y$ on $[0, \pi]$, and set $\alpha(0)=1$ and $\alpha(x)=0$ if $0<x \leq \pi$. It is easy to check that $\alpha$ is a subfunction on $[0, \pi]$. Now let $x_{1}=0, x_{2}=\pi$ and $y(x)=-\sin x$ on $[0, \pi]$. Thus $y(0)=0=\alpha\left(0^{+}\right)$and $y(\pi)=\alpha(\pi)$; however $y(x)<\alpha(x)$ on $(0, \pi)$.

If solutions of two point boundary value problems for (1.1) are unique when they exist then $\Psi$ is a subfunction as can be seen from Klassen [10, Corollary 7] and a simple limiting argument. If $c<b, \alpha$ is bounded and $\mathscr{D} \mathscr{R} \alpha(c)$ is finite then $\psi$ is a subfunction on $[c, d]$ for $0<d-c$ sufficiently small, by Corollary 2.3 .

3. The main results. The following theorem on the continuity of $\mathscr{D} \mathscr{R} \alpha(x)$ is the key result needed in the proof of the differentiability almost everywhere of $\mathscr{D} \mathscr{R} \alpha(x)$.

THEOREM 3.1. Let $\alpha$ be a bounded subfunction for $y^{\prime \prime}=f\left(x, y, y^{\prime}\right)$ on $[a, b]$. If $c \in[a, b)$ and $-\infty<\mathscr{D R} \alpha(c) \leq \infty$ then $\mathscr{D} \mathscr{L} \alpha\left(c^{+}\right)=$ $\mathscr{D} \mathscr{R} \alpha(c)=\mathscr{D} \mathscr{R} \alpha\left(c^{+}\right)$. If $c \in(a, b]$ and $-\infty \leq \mathscr{D} \mathscr{L} \alpha(c)<\infty$ then $\mathscr{D} \mathscr{L} \alpha\left(c^{-}\right)=\mathscr{D} \mathscr{L} \alpha(c)=\mathscr{D} \mathscr{R} \alpha\left(c^{-}\right)$.

Proof. We consider the right continuity of $\mathscr{D R} \alpha(x)$ at $x=c$. The proof of left continuity of $\mathscr{D} \mathscr{L} \alpha(x)$ is similar.

Assume first that $\mathscr{D} \mathscr{R} \alpha(c)=\infty$. Suppose $\liminf _{x \rightarrow c^{+}} \mathscr{D} \mathscr{R} \alpha(x)=$ $L<\infty$. As indicated we may assume that $c=0=\alpha\left(0^{+}\right)$and $L<-1$. Choose $\gamma \in(0, \min \{\eta(1,1,1), 1\} / 2)$ such that $\gamma<\alpha\left(\gamma^{+}\right)<1$ and 
$\zeta \in(\gamma, 2 \gamma)$ such that $\left(\alpha(\zeta)-\alpha\left(\gamma^{+}\right)\right) /(\zeta-\gamma)<-1$ and $\alpha(\zeta)>0$. By Theorem 2.1 the boundary value problem (1.1) with $y(0)=\alpha(\zeta)=$ $y(\zeta)$ has a solution with $\left|y^{\prime}(x)\right|<1$ on $[0, \zeta]$. Thus $y(\gamma)<\alpha\left(\gamma^{+}\right)$. Since $y(0) \geq \alpha\left(0^{+}\right)$it follows from Corollary 2.3 that $y(x) \geq \alpha(x)$ on $(0, \zeta]$, a contradiction. In the case $\liminf _{x \rightarrow 0^{+}} \mathscr{D} \mathscr{L} \alpha(x)=L<-1$ choose $\gamma \in(0, \min \{\eta(1,1,1), 1\})$ such that $\gamma<\alpha\left(\gamma^{-}\right)<1$ and let $y$ be a solution of (1.1), $y(0)=\alpha\left(\gamma^{-}\right)=y(\gamma)$ such that $y(x) \geq \alpha(x)$ on $(0, \gamma)$ and $\left|y^{\prime}(x)\right|<1$ on $[0, \gamma]$. Again we have a contradiction and the result follows.

Consider the case $|\mathscr{D} \mathscr{R} \alpha(c)|<\infty$. As indicated we may assume that $c=0=\alpha\left(0^{+}\right)=\mathscr{D} \mathscr{R} \alpha(0)$. Since $\mathscr{D} \mathscr{L} \alpha(x) \leq \mathscr{D} \mathscr{R} \alpha(x)$, it suffices to show that

$$
\limsup _{x \rightarrow 0^{+}} \mathscr{D} \mathscr{R} \alpha(x) \leq 0 \leq \liminf _{x \rightarrow 0^{+}} \mathscr{D} \mathscr{L} \alpha(x) .
$$

Given $\varepsilon>0$ choose $\delta=\delta(\varepsilon)>0$ such that $\delta \leq b$ and

$$
|\alpha(x)|<\varepsilon x \text {, for all } x \in(0, \delta) \text {. }
$$

Assume the left hand inequality in (3.1) does not hold. Then there exists $\varepsilon>0$ such that $\varepsilon<\frac{1}{2}$ and

$$
\limsup _{x \rightarrow 0+} \mathscr{D} \mathscr{R} \alpha(x)>4 \varepsilon \text {. }
$$

Let $K=\min (\delta, \eta)$, where $\eta=\eta(1,1, \varepsilon)$. Choose $x_{2} \in(0, K)$ and let $y_{2}=\alpha\left(x_{2}\right)$. By (3.2) there is $\theta$ such that $0<\theta<x_{2}$ and

$$
\left|\frac{\alpha\left(x_{2}\right)-\alpha(x)}{x_{2}-x}\right|<2 \varepsilon \quad \text { for all } x \in(0, \theta) \text {. }
$$

Choose $x_{1} \in(0, \theta)$ such that

$$
\mathscr{D} \mathscr{R} \alpha\left(x_{1}\right)>3 \varepsilon .
$$

Let $y_{1}=\alpha\left(x_{1}^{+}\right)$so that $\left|\left(y_{2}-y_{1}\right) /\left(x_{2}-x_{1}\right)\right|<2 \varepsilon$, by (3.4). By Corollary 2.3 there is a solution $y$ on $\left[x_{1}, x_{2}\right]$ such that $y\left(x_{1}\right)=y_{1}, y\left(x_{2}\right)=$ $y_{2}, y(x) \geq \alpha(x)$ and $\left|y^{\prime}(x)-\left(y_{2}-y_{1}\right) /\left(x_{2}-x_{1}\right)\right|<\varepsilon$ on $\left(x_{1}, x_{2}\right)$. Thus $\left|y^{\prime}(x)\right| \leq 3 \varepsilon$ on $\left[x_{1}, x_{2}\right]$, but $\alpha\left(x_{1}^{+}\right)=y\left(x_{1}\right)$ and $y^{\prime}\left(x_{1}\right) \leq 3 \varepsilon<$ $\mathscr{D R} \alpha\left(x_{1}\right)$, a contradiction.

Suppose now that the right hand inequality does not hold in (3.1). Choose $\varepsilon$ such that $\frac{1}{2}>\varepsilon>0$ and

$$
\liminf _{x \rightarrow 0^{+}} \mathscr{D} \mathscr{L} \alpha(x)<-3 \varepsilon .
$$

Choose $\eta=\eta(1,1, \varepsilon)$ and $x_{2}$ such that $0<x_{2}<\min (\eta, \delta)$ and $\mathscr{D} \mathscr{L} \alpha\left(x_{2}\right)<-3 \varepsilon$. Let $x_{1}=0, y_{1}=\alpha\left(x_{1}^{+}\right)=0$ and $y_{2}=\alpha\left(x_{2}^{-}\right)$. 
Thus $\left|\left(y_{2}-y_{1}\right) /\left(x_{2}-x_{1}\right)\right|<\varepsilon$ so by an extension of the argument in Corollary 2.3 there exists a solution $y$ on $\left[x_{1}, x_{2}\right]$ satisfying $y(x) \geq \alpha(x)$ and $\left|y^{\prime}(x)-\left(y_{2}-y_{1}\right) /\left(x_{2}-x_{1}\right)\right|<\varepsilon$ on $\left(x_{1}, x_{2}\right)$. Thus $y^{\prime}\left(x_{2}\right) \geq-2 \varepsilon>$ $\mathscr{D} \mathscr{L} \alpha\left(x_{2}\right)$, a contradiction. Thus (3.1) holds and the proof is complete.

THEOREM 3.2. Let $\alpha$ be a bounded subfunction for $y^{\prime \prime}=f\left(x, y, y^{\prime}\right)$ on $[a, b]$. If $c \in[a, b)$ and $|\mathscr{D} \mathscr{R} \alpha(c)|<\infty$, then

$$
D_{+} \mathscr{D} \mathscr{R} \alpha(c) \geq f\left(c, \alpha\left(c^{+}\right), \mathscr{D} \mathscr{R} \alpha(c)\right) \text {. }
$$

Proof. By Theorem 3.1 and Theorem 2.5 there is $\delta>0$ such that $\alpha$ is continuous and $\mathscr{D} \mathscr{R} \alpha(c)+1 \geq \mathscr{D} \mathscr{R} \alpha(x) \geq \mathscr{D} \mathscr{L} \alpha(x) \geq \mathscr{D} \mathscr{R} \alpha(c)-1$ on $(c, c+\delta)$. Thus it suffices to show that

$$
\liminf _{x \rightarrow c^{+}} \frac{\mathscr{D} \mathscr{L} \alpha(x)-\mathscr{D} \mathscr{R} \alpha(c)}{x-c} \geq f\left(c, \alpha\left(c^{+}\right), \mathscr{D} \mathscr{R} \alpha(c)\right) .
$$

As $|\mathscr{D} \mathscr{R} \alpha(c)|<\infty$ we see from the proof of Theorem 3.1 that given $\varepsilon>0$ there is $\delta>0$ such that for any $t \in(c, c+\delta)$ there is a solution $y$ on $[c, t]$ satisfying $y(c)=\alpha\left(c^{+}\right), y(t)=\alpha(t), y(x) \geq \alpha(x)$, and $\left|y^{\prime}(x)-\mathscr{D} \mathscr{R} \alpha(c)\right|<\varepsilon$ on $(c, t]$. For these solutions we have

$$
\begin{aligned}
\frac{\mathscr{D} \mathscr{L} \alpha(t)-\mathscr{D} \mathscr{R} \alpha(c)}{t-c} & \geq \frac{y^{\prime}(t)-y^{\prime}(c)}{t-c} \\
& =y^{\prime \prime}(\xi) \text { some } \xi \in(c, t) \\
& =f\left(\xi, y(\xi), y^{\prime}(\xi)\right) .
\end{aligned}
$$

Noting that these solutions satisfy $\left(\xi, y(\xi), y^{\prime}(\xi)\right)$ converges to $\left(c, \alpha\left(c^{+}\right)\right.$, $\mathscr{D} \mathscr{R} \alpha(c))$ as $\varepsilon$ converges to zero the result follows.

REMARK. A similar argument to the above yields the following. If $c \in(a, b]$ and $|\mathscr{D} \mathscr{L} \alpha(c)|<\infty$, then

$$
D_{-} \mathscr{D} \mathscr{L} \alpha(c) \geq f\left(x, \alpha\left(c^{-}\right), \mathscr{D} \mathscr{L} \alpha(c)\right) \text {. }
$$

LEMMA 3.3. Let $\Phi:(c, d] \rightarrow \mathbf{R}$ satisfy $\liminf _{t \rightarrow x^{-}} \mathscr{D R} \Phi(t) \leq$ $\mathscr{D R} \Phi(x)$ and $D_{+} \mathscr{D} \mathscr{R} \Phi(x)>0$ on $(c, d)$. Then $\mathscr{D} \mathscr{R} \Phi(x)$ is nondecreasing on $(c, d]$ and hence differentiable almost everywhere on $(c, d)$.

Proof. Suppose $\mathscr{D} \mathscr{R} \Phi(x)$ is not non-decreasing on $(c, d]$. Then there exist $u, v$ with $c>u>v \leq d$ and $\mathscr{D} \mathscr{R} \Phi(u)>\mathscr{D} \mathscr{R} \Phi(v)$. Let

$$
t=\sup \{s \in[u, v]: \mathscr{D} \mathscr{R} \Phi(x) \geq \mathscr{D} \mathscr{R} \Phi(u) \text { for all } x \in[u, s]\} .
$$

If $u<t \leq v$ then $\mathscr{D} \mathscr{R} \Phi(t) \geq \liminf _{x \rightarrow t^{-}} \mathscr{D R} \Phi(x) \geq \mathscr{D R} \Phi(u)$. Thus $u \leq t<v$ and $\mathscr{D} \mathscr{R} \Phi(t) \geq \mathscr{D} \mathscr{R} \Phi(u)$. From the definition of $t$ it follows 
that $D_{+} \mathscr{D} \mathscr{R} \Phi(t) \leq 0$, a contradiction, and hence $\mathscr{D} \mathscr{R} \Phi(u) \leq \mathscr{D} \mathscr{R} \Phi(v)$ for all $c<u<v \leq d$, as required.

The following lemma is needed in Theorems $3.1^{\prime}$ and 3.10 and the examples in the next section. It is of interest in its own right and will be used in a forthcoming paper where we will use subfunctions and superfunctions to prove existence of solutions, and discuss the relationship between the various definitions of subfunctions and lower solutions.

Let $\alpha:[a, b] \rightarrow \mathbf{R}$. Recall that $\alpha\left(a^{-}\right)=\alpha(a)$ and $\alpha\left(b^{+}\right)=\alpha(b)$. We will make the following assumption about $\alpha$.

Assumption 3.4. The function $\alpha$ satisfies the following:

(i) $\alpha\left(x^{\mp}\right)$ exist in the reals for all $x \in[a, b]$;

(ii) $\alpha(x) \leq \max \left\{\alpha\left(x^{+}\right), \alpha\left(x^{-}\right)\right\}$;

(iii) $\mathscr{D} \mathscr{R} \alpha(x)$ and $\mathscr{D} \mathscr{L} \alpha(x)$ exist in the extended reals for all $x \in$ $[a, b)$ and $(a, b]$, respectively;

(iv) $\mathscr{D} \mathscr{R} \alpha(x) \geq \mathscr{D} \mathscr{L} \alpha(x)$ if $\alpha$ is continuous at $x \in(a, b)$; and

(v) $\mathscr{D} \mathscr{L} \alpha(x)=-\infty$, if $\alpha\left(x^{-}\right)>\min \left\{\alpha(x), \alpha\left(x^{+}\right)\right\}$while $\mathscr{D} \mathscr{R} \alpha(x)=$ $\infty$, if $\alpha\left(x^{+}\right)>\min \left\{\alpha(x), \alpha\left(x^{-}\right)\right\}$, for all $x \in(a, b]$ and $[a, b)$, respectively.

Lemma 3.5. Let $\alpha:[a, b] \rightarrow \mathbf{R}$ be bounded and satisfy Assumption 3.4. If $\left[x_{1}, x_{2}\right] \subset[a, b], y \in C^{1}[a, b], y\left(x_{i}\right) \geq \alpha\left(x_{i}\right)$ for $i=1,2$, and $l=\sup \left\{\alpha(x)-y(x): x_{1} \leq x \leq x_{2}\right\}>0$, then there exists $c \in\left(x_{1}, x_{2}\right)$ such that $\alpha(c)-y(c)=l, \alpha^{\prime}(c)$ exists and $\alpha^{\prime}(c)=y^{\prime}(c)$.

Proof. Let $c \in\left[x_{1}, x_{2}\right]$ satisfy

$$
l=\sup \left\{\alpha(x)-y(x): x_{1} \leq x \leq x_{2},|x-c|<\delta\right\},
$$

for all $\delta>0$. If $c=x_{1}$ then $\alpha\left(x_{1}^{+}\right)>\alpha\left(x_{1}\right)$ since $y\left(x_{1}\right) \geq \alpha\left(x_{1}\right)$ and $l>$ 0 . In view of $(\mathrm{v})$ of Assumption 3.4, $\mathscr{D} \mathscr{R} \alpha\left(x_{1}\right)=\infty$ contradicting the definition of $c$. Thus $x_{1}<c$ and similarly $c<x_{2}$. A similar argument shows that $c$ is a point of continuity of $\alpha$. Since $\mathscr{D} \mathscr{R} \alpha(c) \geq \mathscr{D} \mathscr{L} \alpha(c)$, $x_{1}<c<x_{2}$, and $y^{\prime}(c)$ exists, then $\alpha^{\prime}(c)$ exists with $y^{\prime}(c)=\alpha^{\prime}(c)$.

REMARK. Let $\alpha$ satisfy Assumption 3.4, $\left(x_{1}, x_{2}\right)$ be a subinterval of $[a, b], y \in C^{1}\left[x_{1}, x_{2}\right]$ and $l=\sup \left\{\alpha(x)-y(x): x_{1}<x<x_{2}\right\}>0$. The other assumptions in Lemma 3.5 serve to guarantee that $l=$ $\sup \{\alpha(x)-y(x): d \leq x \leq e\}$ for some $d, e \in\left(x_{1}, x_{2}\right)$. From this observation we can obtain variants of Lemma 3.5. For example, assume instead of these other assumptions that $\alpha\left(x_{2}\right)=y\left(x_{2}\right), \alpha^{\prime}\left(x_{2}\right)$ exists, 
$\alpha^{\prime}\left(x_{2}\right)<y^{\prime}\left(x_{2}\right)$ and $\mathscr{D} \mathscr{R} \alpha\left(x_{1}\right)=\infty$. Then there exists $c \in\left(x_{1}, x_{2}\right)$ such that $\alpha(c)-y(c)=l, \alpha^{\prime}(c)$ exists and $\alpha^{\prime}(c)=y^{\prime}(c)$. We need such a variant in the proof of Theorem 3.12.

Now we have the background to extend Theorem 3.1 .

THEOREM 3.1'. Under the assumptions of Theorem 3.1, $\mathscr{D} \mathscr{L} \alpha\left(c^{+}\right)=$ $\mathscr{D} \mathscr{R} \alpha(c)=\mathscr{D} \mathscr{R} \alpha\left(c^{+}\right)$, for all $c \in[a, b)$ and $\mathscr{D} \mathscr{R} \alpha\left(c^{-}\right)=\mathscr{D} \mathscr{L} \alpha(c)=$ $\mathscr{D} \mathscr{L} \alpha\left(c^{-}\right)$, for all $c \in(a, b]$.

Proof. We prove that $\mathscr{D} \mathscr{R} \alpha\left(c^{-}\right)=\mathscr{D} \mathscr{R} \alpha(c)=\mathscr{D} \mathscr{L} \alpha\left(c^{-}\right)$, for $c \in$ $(a, b]$; the proof of the other part is similar. In view of Theorem 3.1 it suffices to consider the case $\mathscr{D} \mathscr{L} \alpha(c)=\infty$. Since $\mathscr{D} \mathscr{L} \alpha\left(t^{+}\right)=\mathscr{D} \mathscr{R} \alpha(t)$ when $\mathscr{D} \mathscr{R} \alpha(t)>-\infty$, by Theorem 3.1, then $\mathscr{D} \mathscr{L} \alpha\left(c^{-}\right)=\mathscr{D} \mathscr{L} \alpha(c)=\infty$ if $\mathscr{D R} \alpha\left(c^{-}\right)=\mathscr{D} \mathscr{L} \alpha(c)=\infty$. Thus it suffices to show this latter equality. From Theorem 3.2 there is a nonincreasing function $b:[0, \infty) \rightarrow$ $(-\infty, 0)$ such that $D_{+} \mathscr{D} \mathscr{R} \alpha(x)>b(n)$ whenever $|\mathscr{D} \mathscr{R} \alpha(x)| \leq n$ and $x \in[a, b)$.

First we show the following. If $x_{1} \in[a, b), \mathscr{D} \mathscr{R} \alpha\left(x_{1}\right) \geq n$ and $\kappa=|b(n)|^{-1}$ then $\mathscr{D} \mathscr{R} \alpha(x) \geq n-1$, for $x \in\left[x_{1}, x_{1}+\kappa\right] \cap[a, b)$. To see this we suppose that $\mathscr{D} \mathscr{R} \alpha(s)<n-1$, for some $s \in\left[x_{1}, x_{1}+\kappa\right] \cap\left[x_{1}, b\right)$, choose $\left[x_{2}, x_{3}\right] \subset\left[x_{1}, x_{1}+\kappa\right] \cap[a, b)$ such that $\mathscr{D} \mathscr{R} \alpha(x) \leq n$, for all $x \in\left[x_{2}, x_{3}\right], \mathscr{D} \mathscr{R} \alpha(x) \geq n-1$, for all $x \in\left[x_{1}, x_{3}\right], \mathscr{D} \mathscr{R} \alpha\left(x_{2}\right)=n$ and $\mathscr{D R} \alpha\left(x_{3}\right)=n-1$, and show that $x_{3}-x_{2} \geq \kappa$. We choose $x_{2}$ and $x_{3}$ as follows. Let $x_{3}=\inf \left\{t \in\left[x_{1}, s\right]: \mathscr{D} \mathscr{R} \alpha(t) \leq n-1\right\}$. Thus $\mathscr{D} \mathscr{R} \alpha(t)>$ $n-1$, for all $t \in\left[x_{1}, x_{3}\right)$. As $\mathscr{D} \mathscr{R} \alpha\left(x_{1}^{+}\right)=\mathscr{D} \mathscr{R} \alpha\left(x_{1}\right) \geq n$, then $x_{3}>x_{1}$. If $\mathscr{D} \mathscr{R} \alpha\left(x_{3}\right)<n-1$, then $\mathscr{D} \mathscr{R} \alpha\left(x_{3}^{-}\right)=\mathscr{D} \mathscr{L} \alpha\left(x_{3}\right) \leq \mathscr{D} \mathscr{R} \alpha\left(x_{3}\right)<n-1$ and there exists $t \in\left[x_{1}, x_{3}\right)$ such that $\mathscr{D} \mathscr{R} \alpha(t)<n-1$, a contradiction. If $\mathscr{D} \mathscr{R} \alpha\left(x_{3}\right)>n-1$ then there is $\eta>0$ such that $\mathscr{D} \mathscr{R} \alpha(t)>n-1$, for all $t \in\left[x_{3}, x_{3}+\eta\right]$, a contradiction to the definition of $x_{3}$. Thus $\mathscr{D} \mathscr{R} \alpha\left(x_{3}\right)=n-1$. Let $x_{2}=\sup \left\{t \in\left[x_{1}, x_{3}\right]: \mathscr{D} \mathscr{R} \alpha(t) \geq n\right\}$. By a similar argument to the above we see that $\mathscr{D} \mathscr{R} \alpha\left(x_{2}\right)=n$. We see that $\kappa \leq x_{3}-x_{2} \leq x_{3}-x_{1}$ as follows. We note that $\mathscr{D} \mathscr{L} \alpha(x) \leq \mathscr{D} \mathscr{R} \alpha(x) \leq n$ and then $\mathscr{D} \mathscr{L} \alpha(x)>-\infty$, for all $x \in\left(x_{2}, x_{3}\right]$, otherwise $\mathscr{D} \mathscr{R} \alpha\left(x^{-}\right)=$ $\mathscr{D} \mathscr{L} \alpha(x)=-\infty$ and $\mathscr{D} \mathscr{R} \alpha(t)<n-1$, some $x_{2}<t<x \leq x_{3}$. Thus $\alpha$ is continuous on $\left[x_{2}, x_{3}\right]$ and $D_{+} \mathscr{D} \mathscr{R} \alpha(x)>b(n)$ on $\left(x_{2}, x_{3}\right]$. By Lemma 3.3, $\mathscr{D} \mathscr{R} \alpha(x)-b(n) x$ is strictly increasing on $\left(x_{2}, x_{3}\right]$ and since $\mathscr{D} \mathscr{R} \alpha\left(x_{2}\right)=\mathscr{D} \mathscr{R} \alpha\left(x_{2}^{+}\right)$it follows that $|b(n)|\left(x_{3}-x_{2}\right)>1$, the required contradiction.

Given $\varepsilon>0$ and $t \in[a, b)$ we show that there exists $\xi \in[t, c)$ such that $\mathscr{D} \mathscr{R} \alpha(\xi) \geq k(\varepsilon)$ where $k(\varepsilon)=\left(\alpha\left(c^{-}\right)-\alpha(t)\right) /(c-t)-\varepsilon$. To see this 
let $y(x)=\alpha(t)+k(\varepsilon)(x-t)$. Thus $y(t)=\alpha(t)$ and $y(c)<\alpha\left(c^{-}\right)$. Either $\mathscr{D R} \alpha(t) \geq k(\varepsilon)$ and we may set $\xi=t$ or $0<l=\sup \{\alpha(x)-y(x): t \leq$ $x<c\}$. Assume $l>0$. By Lemma 3.5, there exists $\xi \in(t, c)$ such that $\alpha^{\prime}(\xi)=y^{\prime}(\xi)=k(\varepsilon)$, as required.

From the above results given $n>0$ choosing $\varepsilon=1$ and $t \in$ $\left(c-|b(n+1)|^{-1}, c\right)$ such that $\left(\alpha\left(c^{-}\right)-\alpha(t)\right) /(c-t) \geq n+2$ there is $\xi \in[t, c)$ such that $\mathscr{D} \mathscr{R} \alpha(\xi) \geq n+1$ and $\mathscr{D} \mathscr{R} \alpha(x) \geq n$, for all $x \in[\xi, c)$, as required.

The next result gives sharp smoothness results for a subfunction.

We remind the reader of the convention by which $z\left(a^{-}\right)=z(a)$, $z\left(b^{+}\right)=z(b), \mathscr{D} \mathscr{L} z(a)=\mathscr{D} \mathscr{R} z(a)$ and $\mathscr{D} \mathscr{R} z(b)=\mathscr{D} \mathscr{L} z(b)$. This will be used in the next few results.

THEOREM 3.6. Let $\alpha$ be a bounded subfunction for $y^{\prime \prime}=f\left(x, y, y^{\prime}\right)$ on $[a, b]$. There exists a relatively open set $O$ of $[a, b]$ with measure $0=b-a$ such that on $O, \alpha$ is continuous, $\alpha^{\prime}$ exists in the reals, except possibly for a countable number of points, $\mathscr{D L} \alpha \leq \mathscr{D} \mathscr{R} \alpha$ exist in the reals everywhere and are differentiable almost everywhere.

Proof. From Theorem 2.7 setting $k(x)=\max \{|\mathscr{D} \mathscr{R} \alpha(x)|,|\mathscr{D} \mathscr{L} \alpha(x)|\}$ and $M=\{x \in[a, b]: k(x)=\infty\}$ it follows that $|M|=0$. If $c \in$ $(a, b) \backslash M$, then it follows from Theorems 2.5 and 3.1 and Lemma 2.7 that there is $\delta>0$ such that

$$
-k(c)-1<\mathscr{D} \mathscr{L} \alpha(x) \leq \mathscr{D} \mathscr{R} \alpha(x)<k(c)+1
$$

and $\alpha$ is continuous on $(c-\delta, c+\delta) \subset(a, b)$. Thus there is a relatively open set $O \subset[a, b]$ such that $|[a, b] \backslash O|=0, \alpha$ is continuous on $O, \alpha^{\prime}$ exists in the reals except possibly for a countable set of points $N \subset$ $O$, and $k(x)=\infty$, for all $x \in[a, b] \backslash O$. Let $[c, d] \subset O$; then by compactness and (3.7) it follows that there is $n>0$ such that $k(x) \leq$ $n$ on $[c, d]$. As in the proof of Theorem $3.1^{\prime}, D_{+} \mathscr{D R} \alpha(x)>b(n)$ on $[c, d]$. Since $\liminf \operatorname{in}_{t \rightarrow x^{-}} \mathscr{D} \mathscr{R} \alpha(t)=\mathscr{D} \mathscr{L} \alpha(x) \leq \mathscr{D} \mathscr{R} \alpha(x)$, setting $\Phi(x)=\alpha(x)-b(n) x^{2} / 2$ in Lemma 3.3 we see that $\mathscr{D} \mathscr{R} \Phi$ and hence $\mathscr{D} \mathscr{R} \alpha$ is differentiable almost everywhere in $(c, d)$. The differentiability almost everywhere of $\mathscr{D} \mathscr{L} \alpha$ follows by a similar argument.

We show in Example 4.3 that Theorem 3.6 gives the best possible smoothness properties.

We now consider the smoothness properties of a function which is simultaneously a subfunction and a superfunction. We show that the 
assumption that two point boundary value problems have at most one solution can be deleted from [9, Theorem 4.10].

THEOREM 3.7. Let $z$ be bounded and simultaneously a subfunction and a superfunction for $y^{\prime \prime}=f\left(x, y, y^{\prime}\right)$ on $[a, b]$. Then there is $a$ relatively open subset $O$ of $[a, b]$ of full measure such that, on each component $\mathscr{C}$ of $O, z$ is a solution on its maximal interval of existence. Let $c \in[a, b] \backslash O$. If $c$ is a point of continuity of $z$ then either $z^{\prime}(c)=\infty$ or $z^{\prime}(c)=-\infty$. If $z\left(c^{-}\right)>z\left(c^{+}\right)$then $\mathscr{D} \mathscr{L} z(c)=-\infty=$ $\mathscr{D} \mathscr{R} z(c)$. If $z\left(c^{-}\right)<z\left(c^{+}\right)$then $\mathscr{D} \mathscr{L} z(c)=\infty=\mathscr{D} \mathscr{R} z(c)$. Moreover $\min \left\{z\left(c^{-}\right), z\left(c^{+}\right)\right\} \leq z(c) \leq \max \left\{z\left(x^{-}\right), z\left(c^{+}\right)\right\}$.

Proof. Let $k(x), M$, and $O$ be as given in the proof of Theorem 3.6. Let $c \in M=[a, b] \backslash O$. Thus $k(c)=\infty$. If $c$ is a point of continuity of $z$ we see from Lemma 2.6 and its analogue for superfunctions that $z^{\prime}(c)$ exists and $\left|z^{\prime}(c)\right|=k(c)=\infty$. If $a<c<b$ and $z\left(c^{-}\right)>z\left(c^{+}\right)$ we see from Theorem 2.5 that $\mathscr{D} \mathscr{L} z(c)=-\infty$ and from its analogue for superfunctions that $\mathscr{D} \mathscr{R} z(c)=-\infty$. Similarly if $a<c<b$ and $z\left(c^{-}\right)<z\left(c^{+}\right)$then $\mathscr{D} \mathscr{L} z(c)=\infty=\mathscr{D} \mathscr{R} z(c)$. The cases $c \in\{a, b\}$ is a point of discontinuity follow similarly.

From Theorem 3.6 and its analogue for superfunctions we see that $z^{\prime}(x)$ exists and is real valued for all $x \in O$. From Theorem 3.1', $z^{\prime}$ is continuous on $O$ and by Theorem 3.2, (3.5), and the corresponding results for superfunctions $z^{\prime \prime}=f\left(x, z, z^{\prime}\right)$, almost everywhere in $O$. From the continuity of $f\left(x, z, z^{\prime}\right), z^{\prime \prime}=f\left(x, z, z^{\prime}\right)$, for all $x \in O$. Let $\mathscr{C}$ be a component of $O$ with $\overline{\mathscr{C}}=[d, e]$. If $d \notin \mathscr{C}$, then $|\mathscr{D} \mathscr{R} z(d)|=\infty$ and $z^{\prime}\left(d^{+}\right)=\mathscr{D} \mathscr{R} z(d)$, by Theorem 3.1'. If $d \in \mathscr{C}$, then $d=a$, $|\mathscr{D} \mathscr{R} z(a)|<\infty$, and $z \in C^{2}[a, e)$. A similar argument applies at $e$. Thus $\mathscr{C}$ is a maximal interval of existence for the solution $z$. (See [8].) The last part follows from Theorem 2.4 and its analogue for superfunctions.

Example 4.1 shows that Theorem 3.7 gives the best smoothness for $z$.

We now introduce the definition of the local unique boundary value property and use it to show that the function $z$ given by (1.7) is a solution on an open set of full measure. We also give sufficient conditions on $f$ in order that (1.1) has this property.

Definition 3.8. We say (1.1) has the local unique boundary value property on $[a, b]$ if to each $c \in(a, b), M>0$ and $N>0$ there exists 
$\delta=\delta(c, M, N)>0$ such that there is at most one solution of (1.1) and (1.2) satisfying $\left|\left(y_{2}-y_{1}\right) /\left(x_{2}-x_{1}\right)\right| \leq N$ and $|y(x)| \leq M$ for all $a \leq c-\delta<x_{1} \leq x_{2}<c+\delta \leq b$.

To require that (1.1) has the local unique boundary value property is weaker than requiring $f$ is such that all two point boundary value problems have at most one solution.

The local unique boundary value property will be satisfied if $f$ is continuous on $[a, b] \times \mathbf{R}^{2}$ and locally lipschitz with respect to $\left(y, y^{\prime}\right)$. This can be seen from the following observations. If $y$ is any solution satisfying $\left|\left(y_{2}-y_{2}\right) /\left(x_{2}-x_{1}\right)\right| \leq N$ and $|y(x)| \leq M$ on $\left[x_{1}, x_{2}\right]$ there is $\xi \in\left(x_{1}, x_{2}\right)$ with $\left|y^{\prime}(\xi)\right| \leq N,|y(\xi)| \leq M$. By Theorem 2.2, setting $\kappa=\kappa(M, N, 1)=(Q+N+1)^{-1}, y$ exists and satisfies $\left|y^{\prime}(x)\right| \leq N+1$ on $[\xi-\kappa, \xi+\kappa] \cap[a, b]$. Suppose that

$$
\left|f\left(x, y_{1}, p_{1}\right)-f\left(x, y_{2}, p_{2}\right)\right| \leq K(M, N)\left|y_{1}-y_{2}\right|+L(M, N)\left|p_{1}-p_{2}\right|
$$

for $\left|y_{1}\right|,\left|y_{2}\right| \leq M,\left|p_{1}\right|,\left|p_{2}\right| \leq N+1$ and all $x \in[a, b]$ then choosing $\delta(c, M, N)=\min \{\kappa, 1 /(k+L)\} / 2$ there is at most one solution satisfying $\left|y^{\prime}(x)\right| \leq N+1,|y(x)| \leq M$ and $\left|\left(y_{2}-y_{1}\right) /\left(x_{2}-x_{1}\right)\right| \leq N$ on $\left[x_{1}, x_{2}\right]$. This follows immediately from well-known results. See, for example, Bailey, Waltman and Shampine [3].

From the above $f$ has the local unique boundary value property if it has continuous partial derivatives.

Example 4.2 of the next section shows that the local uniqueness property is not sufficient for the proof [9, Theorem 4.12]; that is, to prove that $z$ given by (1.7) is a superfunction.

THEOREM 3.9. Let $\alpha$ and $\beta$ be bounded subfunctions and superfunctions, respectively, for $y^{\prime \prime}=f\left(x, y, y^{\prime}\right)$ on $[a, b]$ satisfying $\alpha(x) \leq \beta(x)$ on $[a, b]$. Let $f$ have the local unique boundary value property and $z$ be given by (1.7). Then there is a relatively open subset $O$ of $[a, b]$ of full measure such that on each component $\mathscr{C}$ of $O, z$ is a solution on its maximal interval of existence. If $c \in[a, b] \backslash O$ is a point of continuity of $z$ then either $z^{\prime}(c)=\infty$ or $z^{\prime}(c)=-\infty$. If $z\left(c^{-}\right)>z\left(c^{+}\right)$then $\mathscr{D} \mathscr{L} z(c)=-\infty=\mathscr{D} \mathscr{R} z(c)$. If $z\left(c^{+}\right)>z\left(c^{-}\right)$then $\mathscr{D} \mathscr{L} z(c)=\infty=$ $\mathscr{D} \mathscr{R} z(c)$. Moreover $\min \left\{z\left(c^{-}\right), z\left(c^{+}\right)\right\} \leq z(c) \leq \max \left\{z\left(c^{-}\right), z\left(c^{+}\right)\right\}$.

Proof. By [9, Theorem 4.6], $z$ is a subfunction. Let $O$ and $k(x)$ be as given in the proof of Theorem 3.6. Then $\infty>\mathscr{D} \mathscr{R} z(x) \geq \mathscr{D} \mathscr{L} z(x)>$ $-\infty$, for all $x \in O$.

First we show that $z$ is a solution on $O$. Let $c \in O \cap(a, b)$. Consider the boundary value problem $y^{\prime \prime}=f\left(x, y, y^{\prime}\right), y(c \pm \delta)=z(c \pm \delta)$, where 
$0<\delta \leq \min \{b-c, c-a\}$ is chosen below. Any solution $v$ of these problems will satisfy $\alpha(x) \leq z(x) \leq v(x) \leq \beta(x)$ and hence $|v(x)| \leq M=$ $\sup \{|\alpha(x)|,|\beta(x)|: a \leq x \leq b\}$. Setting $N=|\mathscr{D} \mathscr{R} z(c)|+|\mathscr{D} \mathscr{L} z(c)|+1$ and $\varepsilon=1$ in Theorem 2.1, $N=|\mathscr{D} \mathscr{R} z(c)|+|\mathscr{D} \mathscr{L} z(c)|+2$ in Definition 3.8 and choosing $\delta<\min \{\gamma, \delta(c, M, N)\} / 2$ there is a unique solution $u$ to the above problem. Moreover $u$ is the unique solution of $y^{\prime \prime}=$ $f\left(x, y, y^{\prime}\right), y\left(x_{i}\right)=u\left(x_{i}\right)$, for $i=1,2$, and any $\left[x_{1}, x_{2}\right] \subset[c-\delta, c+\delta]$. Set

$$
w(x)= \begin{cases}z(x), & \text { if } x \in[a, b] \backslash[c-\delta, c+\delta], \\ u(x), & \text { if } x \in[c-\delta, c+\delta] .\end{cases}
$$

As $u$ is a subfunction on $[c-\delta, c+\delta]$ and $u(c \pm \delta) \geq z(c \pm \delta)$, by [9, Theorem 4.7] $w$ is a subfunction on $[a, b]$. Thus $w(x) \equiv z(x)$ on $[a, b]$ and $z$ is a solution on $(c-\delta, c+\delta)$. In the case $c=a$, a simple modification of the above argument shows that $z$ is a solution on $[a, a+\delta)$. The case $c=b$ is similar. Thus $z$ is a solution on $O$.

We consider now the behaviour of $z$ at points in $[a, b] \backslash O$.

Let $c \in[a, b] \backslash O$ be a point of continuity of $z$; then $\mathscr{D R} z(c) \geq$ $\mathscr{D} \mathscr{L} z(c)$ and $k(c)=\infty$. Either $z^{\prime}(c)$ exists and $\left|z^{\prime}(c)\right|=\infty$ or $\infty>$ $\mathscr{D} \mathscr{R} z(c)>\mathscr{D} \mathscr{L} z(c)=-\infty$ or $\infty=\mathscr{D} \mathscr{R} z(c)>\mathscr{D} \mathscr{L} z(c)>-\infty$. Suppose $\infty>\mathscr{D} \mathscr{R} z(c)>\mathscr{D} \mathscr{L} z(c)=-\infty$; then given $\delta>0$ sufficiently small there is $k>0$ such that $k \leq \delta$ and

$$
|(z(c+\delta)-z(c-k)) /(\delta+k)-\mathscr{D} \mathscr{R} z(c)| \leq 1 .
$$

Let $u$ be a solution on $[c-k, c+\delta]$ satisfying $u(c-k)=z(c-k)$ and $u(c+\delta)=z(c+\delta)$ and construct $w$ as in the first part of the proof to obtain the contradiction that $z^{\prime}(c) \in \mathbf{R}$ exists. Similarly we may show $\infty=\mathscr{D} \mathscr{R} z(c)>\mathscr{D} \mathscr{L} z(c)>-\infty$ is impossible. Hence $z^{\prime}(c)$ exists and $\left|z^{\prime}(c)\right|=\infty$.

Let $c \in[a, b] \backslash O$ be a point of discontinuity of $z$. First we show that $\min \left\{z\left(c^{-}\right), z\left(c^{+}\right)\right\} \leq z(c) \leq \max \left\{z\left(c^{-}\right), z\left(c^{+}\right)\right\}$. Now $z(c) \leq$ $\max \left\{z\left(c^{+}\right), z\left(c^{-}\right)\right\}$since $z$ is a subfunction. If $z(c)<\min \left\{z\left(c^{-}\right), z\left(c^{+}\right)\right\}$ then define $\Phi$ by

$$
\Phi(x)= \begin{cases}z(x), & \text { if } x \neq c, \\ \min \left\{z\left(c^{-}\right), z\left(c^{+}\right)\right\}, & \text {if } x=c .\end{cases}
$$

Thus $\Phi$ is a subfunction and $\Phi(x) \leq \beta(x)$, for all $x \in[a, b]$ since $\beta(c) \geq \min \left\{\beta\left(c^{-}\right), \beta\left(c^{+}\right)\right\}$and $\beta(x) \geq z(x)$, for all $x \in[a, b]$. This is a contradiction so $z(c) \geq \min \left\{z\left(c^{-}\right), z\left(c^{+}\right)\right\}$, as required.

Assume that $z\left(c^{+}\right)>z\left(c^{-}\right)$. We show that $\mathscr{D} \mathscr{L} z(c)=\infty=\mathscr{D} \mathscr{R} z(c)$. By Theorem 2.5, $\mathscr{D R} z(c)=\infty$. If $\beta\left(c^{-}\right)=z\left(c^{-}\right)$then $\beta\left(c^{-}\right)<$ $z\left(c^{+}\right) \leq \beta\left(c^{+}\right)$so $\mathscr{D} \mathscr{L} \beta(c)=\infty=\mathscr{D} \mathscr{L} z(c)$, as required. Assume that 
$\beta\left(c^{-}\right)>z\left(c^{-}\right)$; then $\beta(c) \geq \min \left\{\beta\left(c^{-}\right), \beta\left(c^{+}\right)\right\}>\min \left\{z\left(c^{-}\right), z\left(c^{+}\right)\right\}=$ $z\left(c^{-}\right)$. If $-\infty \leq \mathscr{D} \mathscr{L} z(c)<\infty$ then given $\delta>0$ sufficiently small there is $k>0$ such that $0 \leq\left(z\left(c^{-}\right)+k-z(c-\delta)\right) / \delta \leq \max \{\mathscr{D L} z(c), 0\}+1$, and $z\left(c^{-}\right)+k \leq \beta(c)$. Let $u$ be a solution on $[c-\delta, c]$ satisfying $u(c-\delta)=z(c-\delta), u(c)=z\left(c^{-}\right)+k$ and construct $w$ as in the first part of the proof to obtain the contradiction $w(x) \not \equiv z(x)$. Thus $\mathscr{D} \mathscr{L} z(c)=$ $\infty$. A similar argument shows that $\mathscr{D} \mathscr{R} z(c)=-\infty=\mathscr{D} \mathscr{L} z(c)$ when $z\left(c^{-}\right)>z\left(c^{+}\right)$.

Let $\mathscr{C}$ be a component of $O$ such that $\overline{\mathscr{C}}=[d, e]$. If $d \in \mathscr{C}$ then $d=$ $a$ and $z \in c^{2}[a, e)$ is a solution on $[a, e)$. If $d \notin \mathscr{C}$ then $|\mathscr{D} \mathscr{R} z(d)|=\infty$ and by Theorem $3.1^{\prime}, z^{\prime}\left(d^{+}\right)=\mathscr{D} \mathscr{R} z(d)$ so $(d, e)$ is a left maximal interval of existence (see [8]). A similar argument applies at $e$.

REMARKs. If $\Phi$ is a subfunction for (1.1) satisfying $\Phi(a) \leq A$ then

$$
\Phi_{1}(x)= \begin{cases}A, & \text { if } x=a, \\ \Phi(x), & \text { if } a<x \leq b\end{cases}
$$

is a subfunction; hence $z(a)=A$.

In the proof of Theorem 3.9 local uniqueness of boundary value problems is only required when $\alpha\left(x_{i}\right) \leq y_{i} \leq \beta\left(x_{i}\right)$, for $i=1,2$.

Theorem 3.10. Let $\alpha:[a, b] \rightarrow \mathbf{R}$ be bounded. There exists a continuous function $f:[a, b] \times \mathbf{R}^{2} \rightarrow \mathbf{R}$ such that $\alpha$ is a subfunction for $y^{\prime \prime}=f\left(x, y, y^{\prime}\right)$ on $[a, b]$ iff $\alpha$ satisfies Assumption 3.4 and there exists a nonincreasing function $b:[0, \infty) \rightarrow(-\infty, 0]$ such that $D_{+} \mathscr{D} \mathscr{R} \alpha(x)>$ $b(s)$ for all $x \in(a, b)$ and $s \in(0, \infty)$ such that $\alpha$ is continuous at $x$ and $|\mathscr{D} \mathscr{R} \alpha(x)| \leq s$.

Proof. The necessity follows from earlier results so we prove sufficiency. Assume that $|\alpha(x)|<k$, for all $x \in[a, b]$. For $n \in \mathbf{Z}$, the integers, set

$$
\begin{aligned}
A_{n}=\left\{\left(x, y, y^{\prime}\right) \in[a, b] \times \mathbf{R}^{2}: 2 n \leq \alpha^{\prime}(x) \leq 2 n+1,|y| \leq k,\right. \\
\left.2 n \leq y^{\prime} \leq 2 n+1\right\}
\end{aligned}
$$

and

$$
\begin{array}{r}
B_{n}=\left\{\left(x, y, y^{\prime}\right) \in[a, b] \times \mathbf{R}^{2}: 2 n+1 \leq \alpha^{\prime}(x) \leq 2 n+2,|y| \leq k,\right. \\
\left.2 n+1 \leq y^{\prime} \leq 2 n+2\right\} .
\end{array}
$$

Clearly

$$
\bar{A}_{n} \cap\left\{\overline{\bigcup_{m \neq n} A_{m}}\right\}=\varnothing=\bar{B}_{n} \cap\left\{\overline{\bigcup_{m \neq n} B_{m}}\right\},
$$


for all $n \in \mathbf{Z}$. For all $n \in \mathbf{Z}$, let $f_{1}\left(x, y, y^{\prime}\right)=b(2 n+1)$, for all $\left(x, y, y^{\prime}\right) \in \bar{A}_{n}$, and $f_{2}\left(x, y, y^{\prime}\right)=b(2 n+2)$, for all $\left(x, y, y^{\prime}\right) \in \bar{B}_{n}$. Extend $f_{i}$ as continuous functions independent of $y$ to $[a, b] \times[-k, k] \times \mathbf{R}$ and let $f\left(x, y, y^{\prime}\right)=\min \left\{f_{1}\left(x, y, y^{\prime}\right), f_{2}\left(x, y, y^{\prime}\right)\right\}$. Extend $f$ to $[a, b] \times$ $\mathbf{R}^{2}$ as follows. Set

$$
f\left(x, y, y^{\prime}\right)= \begin{cases}f\left(x, k, y^{\prime}\right), & \text { if } y \geq k, \\ f\left(x,-k, y^{\prime}\right)+y+k, & \text { if } y \leq-k .\end{cases}
$$

Clearly $f$ is continuous on $[a, b] \times \mathbf{R}^{2}$. To see that $\alpha$ is a subfunction we proceed as follows. Let $a \leq x_{1}<x_{2} \leq b$ and $y$ be a solution of $y^{\prime \prime}=f\left(x, y, y^{\prime}\right)$ on $\left[x_{1}, x_{2}\right]$ satisfying $y\left(x_{i}\right) \geq \alpha\left(x_{i}\right)$, for $i=1,2$, and assume that $y\left(x_{0}\right)<\alpha\left(x_{0}\right)$, for some $x_{0} \in\left(x_{1}, x_{2}\right)$. By Lemma 3.5, there is $x_{4} \in\left(x_{1}, x_{2}\right)$ such that

$$
\alpha\left(x_{4}\right)-y\left(x_{4}\right)=\sup \left\{\alpha(x)-y(x): x_{1} \leq x \leq x_{2}\right\}
$$

and

$$
\alpha^{\prime}\left(x_{4}\right)=y^{\prime}\left(x_{4}\right) \text {. }
$$

We see that $D_{+} \mathscr{D} \mathscr{R}(\alpha-y)\left(x_{4}\right) \leq 0$ as follows. Either there is $x_{5}$ such that $(\alpha-y)(x)=(\alpha-y)\left(x_{4}\right)$ for $x_{4} \leq x<x_{5} \leq x_{2}$ and $D_{+} \mathscr{D R}(\alpha-y)\left(x_{4}\right)=0$ or there is a strictly decreasing sequence $q_{n}$ convergent to $x_{4}$ such that $(\alpha-y)\left(q_{n}\right)<(\alpha-y)\left(x_{4}\right)$. Moreover, since $(\alpha-y)^{\prime}\left(x_{4}\right)=0$ we may choose $q_{n}$ such that

$$
\left((\alpha-y)\left(q_{n}\right)-(\alpha-y)\left(x_{4}\right)\right) /\left(q_{n}-x_{4}\right)=p_{n},
$$

say, is strictly increasing. Set $w_{n}(x)=(\alpha-y)\left(x_{4}\right)+\left(x-x_{4}\right) p_{n}$ and apply Lemma 3.5 on $\left[x_{4}, q_{n}\right]$ with $\alpha$ replaced by $\alpha-y$ and $y$ replaced by $w_{n}$. Thus there is $c_{n}$ such that $x_{4}<c_{n}<q_{n}$ and $(\alpha-y)^{\prime}\left(c_{n}\right)=w_{n}^{\prime}\left(c_{n}\right)=$ $p_{n}<0$. Thus $D_{+} \mathscr{D R}(\alpha-y)\left(x_{4}\right) \leq 0$. Thus $\left(x_{4}, \alpha\left(x_{4}\right), \alpha^{\prime}\left(x_{4}\right)\right) \in A_{n} \cup B_{n}$ for some $n \in \mathbf{Z}$ and

$$
\begin{aligned}
D_{+} \mathscr{D} \mathscr{R} \alpha\left(x_{4}\right) & >f\left(x_{4}, \alpha\left(x_{4}\right), \alpha^{\prime}\left(x_{4}\right)\right) \\
& \geq f\left(x_{4}, y\left(x_{4}\right), y^{\prime}\left(x_{4}\right)\right)=y^{\prime \prime}\left(x_{4}\right),
\end{aligned}
$$

a contradiction. Thus $\alpha$ is a subfunction as required.

In view of the Theorem 3.9 we immediately obtain the following existence result.

THEOREM 3.11. Let the assumptions of Theorem 3.9 hold and assume that solutions of initial value problems $y^{\prime \prime}=f\left(x, y, y^{\prime}\right), y(c)=y_{0}$, $y^{\prime}(c)=y_{0}^{\prime}$, where $c \in[a, b]$ and $\alpha(c) \leq y_{0} \leq \beta(c)$ can be extended to 
solutions on $[a, b]$. If $\alpha(a) \leq A \leq \beta(a)$ and $\alpha(b) \leq B \leq \beta(b)$, then the two point boundary value problem (1.1) and (1.4) has a solution.

We now apply Perron's method to obtain two existence theorems generalizing [9, Theorems 4.17 and 4.18].

THEOREM 3.12. Let $f, g$ and $h$ be continuous on $[a, b] \times \mathbf{R}^{2}$ and $f$ be such that (1.1) has the local unique boundary property on $[a, b]$. Assume that $g(x, y, p)$ and $h(x, y, p)$ are nondecreasing functions of $y$ and that all solutions of the initial value problems $y(c)=y_{0}, y^{\prime}(c)=y_{0}^{\prime}$, $c \in[a, b]$

$$
y^{\prime \prime}=g\left(x, y, y^{\prime}\right)
$$

and

$$
y^{\prime \prime}=h\left(x, y, y^{\prime}\right)
$$

exist on $[a, b]$. Assume also that for all $y \leq 0$

$$
f(x, y, p)<h(x, y, p)
$$

and

$$
f(x,-y, p)>g(x,-y, p) .
$$

Then the boundary value problem (1.1) and (1.4) has a generalized solution for each $A$ and $B$.

Proof. Let $u$ and $v$ be solutions of the initial value problems $u(a)=$ $u^{\prime}(a)=0=v(a)=v^{\prime}(a),(3.8)$ and (3.9), respectively. Choose $M>0$ sufficiently large that

$$
\alpha(x)=v(x)-M \leq 0 \leq u(x)+M=\beta(x),
$$

on $[a, b]$ and

$$
\alpha(a) \leq A \leq \beta(a), \quad \alpha(b) \leq B \leq \beta(b) .
$$

Thus by (3.8) to (3.12) it follows that

$$
\alpha^{\prime \prime} \geq h\left(x, \alpha, \alpha^{\prime}\right)>f\left(x, \alpha, \alpha^{\prime}\right)
$$

and

$$
\beta^{\prime \prime} \leq g(x, \beta, \beta ;)<f\left(x, \beta, \beta^{\prime}\right) .
$$

Now let $f_{1}:[a, b] \times \mathbf{R}^{2}$ be defined by

$$
f_{1}(x, y, p)= \begin{cases}f(x, \beta(x), p)+y-\beta(x), & \text { if } y \geq \beta(x), \\ f(x, y, p), & \text { if } \alpha(x) \leq y \leq \beta(x), \\ f(x, \alpha(x), p)+y-\alpha(x), & \text { if } y \leq \alpha(x)\end{cases}
$$


By the standard maximum principle argument $\alpha$ and $\beta$ are a subfunction and a superfunction, respectively, for

$$
y^{\prime \prime}=f_{1}\left(x, y, y^{\prime}\right) \text {. }
$$

Now let $z$ be given by

$$
\begin{aligned}
& z(x)=\sup \{\Phi(x): \alpha(t) \leq \Phi(t) \leq \beta(t), \text { for all } t \in[a, b] \\
& \qquad(a) \leq A, \Phi(b) \leq B, \Phi \text { a subfunction for }(3.14)\} .
\end{aligned}
$$

As $f_{1}=f$ for $\alpha(x) \leq y \leq \beta(x)$ and $f$ has the local uniqueness property we see from the remarks following Theorem 3.9 that the conclusions of that theorem hold.

Let $c \in(a, b) \backslash O$. Thus $|\mathscr{D R} z(c)|=\infty$.

Suppose $\mathscr{D} \mathscr{R} z(c)=\infty$. Then $\mathscr{D} \mathscr{L} z(c)=\infty$ and $z\left(c^{-}\right) \leq z\left(c^{+}\right)$so either $z\left(c^{+}\right) \geq 0$ or $z\left(c^{-}\right) \leq 0$. Suppose $z\left(c^{+}\right) \geq 0$. As $\mathscr{D} \mathscr{R} z(c)=\infty$ it follows from Theorem 3.9 and Lemma 3.5 that there is $x_{1} \in(c, b]$ such that $z(x)>0$ for all $x \in\left(c, x_{1}\right]$ and $z^{\prime}\left(x_{1}\right)<\left(z\left(x_{1}\right)-z\left(c^{+}\right)\right) /\left(x_{1}-c\right)$. Now let $w$ be a solution of (3.8) satisfying

$$
w\left(x_{1}\right)=z\left(x_{1}\right), \quad w^{\prime}\left(x_{1}\right)=\left(z\left(x_{1}\right)-z\left(c^{+}\right)\right) /\left(x_{1}-c\right) .
$$

Since $\mathscr{D R} z(c)=\infty, w^{\prime}\left(x_{1}\right)>z^{\prime}\left(x_{1}\right)$ and $z$ is a subfunction for (3.14) on $[a, b]$, from an argument similar to that in Lemma $3.5,0<l=$ $\sup \left\{z(x)-w(x): c<x<x_{1}\right\}=z\left(x_{2}\right)-w\left(x_{2}\right)$ and $z^{\prime}\left(x_{2}\right)=w^{\prime}\left(x_{2}\right)$, for some $x_{2} \in\left(c, x_{1}\right)$. Thus $x_{2} \in O, w\left(x_{2}\right) \leq z\left(x_{2}\right), z\left(x_{2}\right)>0$ and

$$
\begin{aligned}
w^{\prime \prime}\left(x_{2}\right) & =g\left(x_{2}, w\left(x_{2}\right), w^{\prime}\left(x_{2}\right)\right) \leq g\left(x_{2}, z\left(x_{2}\right), w^{\prime}\left(x_{2}\right)\right) \\
& <f\left(x_{2}, z\left(x_{2}\right), z^{\prime}\left(x_{2}\right)\right)=z^{\prime \prime}\left(x_{2}\right),
\end{aligned}
$$

since $\alpha\left(x_{2}\right) \leq z\left(x_{2}\right) \leq \beta\left(x_{2}\right)$, a contradiction.

Suppose $z\left(c^{-}\right) \leq 0$. By Theorem 3.9, Assumptions 3.4 applies to $-z$. Since $\mathscr{D} \mathscr{L} \alpha(c)=\infty$, by an argument similar to that of Lemma 3.5 applied to $-z$, there is $x_{1} \in(a, c)$ such that $z^{\prime}\left(x_{1}\right)<$ $\left(z\left(c^{-}\right)-z\left(x_{1}\right)\right) /\left(c-x_{1}\right)$ and $z(x)<z\left(c^{-}\right)$, for all $x \in\left[x_{1}, c\right)$. Let $w$ be a solution of (3.9) satisfying $w\left(x_{1}\right)=z\left(x_{1}\right)$ and $w^{\prime}\left(x_{1}\right)=$ $\left(z\left(c^{-}\right)-z\left(x_{1}\right)\right) /\left(c-x_{1}\right)$. Since $\mathscr{D} \mathscr{L} z(c)=\infty$ again by a variant of Lemma 3.5 applied to $-z-(-w)$

$$
0<l=\sup \left\{w(x)-z(x): x_{1}<x<c\right\}=w\left(x_{2}\right)-z\left(x_{2}\right)
$$

and $w^{\prime}\left(x_{2}\right)=z^{\prime}\left(x_{2}\right)$, some $x_{2} \in\left(x_{1}, c\right)$. Thus $x_{2} \in O, w\left(x_{2}\right) \geq z\left(x_{2}\right)$, $z\left(x_{2}\right)<0$ and

$$
\begin{aligned}
w^{\prime \prime}\left(x_{2}\right) & =h\left(x_{2}, w\left(x_{2}\right), w^{\prime}\left(x_{2}\right)\right) \geq h\left(x_{2}, z\left(x_{2}\right), z^{\prime}\left(x_{2}\right)\right) \\
& >f\left(x_{2}, z\left(x_{2}\right), z^{\prime}\left(x_{2}\right)\right)=z^{\prime \prime}\left(x_{2}\right),
\end{aligned}
$$

since $\alpha\left(x_{2}\right) \leq z\left(x_{2}\right) \leq \beta\left(x_{2}\right)$, a contradiction. 
A similar argument to the above shows that $\mathscr{D} \mathscr{R} z(c)=-\infty$ is impossible. Thus $O \supset(a, b)$ and $z$ is a generalized solution of (3.15) and (1.4). Since $\alpha(x) \leq z(x) \leq \beta(x)$ it follows that $z$ is a generalized solution of (1.1) and (1.4).

THEOREM 3.13. Let the assumptions of Theorem 3.12 hold and $A=$ $B=0$. Then problem (1.1) and (1.4) has a solution.

Proof. From Theorem 3.12 it suffices to consider $z$ at the end points $a$ and $b$. We consider $x=a$. From Theorem 3.9 either $z\left(a^{+}\right) \geq z(a)=$ 0 and $\mathscr{D R} z(a)=\infty$ or $z\left(a^{+}\right) \leq z(a)=0$ and $\mathscr{D} \mathscr{R} z(a)=-\infty$ or $z \in$ $C^{2}[a, b)$. Now the first two cases lead to a contradiction by arguments similar to those in the proof of Theorem 3.12, so $z \in C^{2}[a, b)$. By a similar argument at $x=b$ we see that $z \in C^{2}[a, b]$ is the required solution.

The assumptions in [9, Theorem 4.17] guarantee all two point boundary value problems (1.1) and (1.2) have at most one solution so (1.1) has the local unique boundary value property. Setting $g(x, y, p)=$ $-k|p|+M$ and $h(x, y, p)=k|p|+M$ we see that [9, Theorem 4.17] follows from our Theorem 3.12.

From an examination of the proof of Theorem 3.12 it is clear that the assumption that the initial value problems for $g$ and $h$ exist on $[a, b]$ can be varied. What is needed in order to produce $\alpha$ and $\beta$ is that (3.8) and (3.9) have at least one solution existing on $[a, b]$, and have solutions $w$ to certain boundary value problems. Under these assumptions it is not clear, for example, that all solutions of the initial value problems (1.1) extend to solutions on $[a, b]$.

REMARK. In Theorem 3.13 we may delete the assumption that $f$ has the local unique boundary value property if we assume that (3.10) and (3.11) hold for all $y$. This may be seen as follows.

Let

$$
E_{n}=\left\{(x, y, p) \in[a, b] \times \mathbf{R}^{2}: n-1 \leq y^{2}+p^{2} \leq n+1\right\}
$$

for $n>0$. Let

$$
c_{n}=\inf \left\{h(x, y, p)-f(x, y, p), f(x, y, p)-g(x, y, p):(x, y, p) \in E_{n}\right\} .
$$

Given $m>0$, using a suitable partition of unity based on the $E_{n}$ 's and Weierstrass's theorem we may approximate $f$ by a continuously differentiable function $f_{m}$ such that $\left|f-f_{m}\right|<1 / m$ everywhere and $\left|f-f_{m}\right|<c_{n}$ on $E_{n}$. Replacing $f$ by $f_{m},(3.10)$ and (3.11) are satisfied 
so by Theorem 3.13 there is a solution $z_{m}$ of (1.1) with $z_{m}(a)=0=$ $z_{m}(b)$. By the mean value theorem there is $x_{m} \in[a, b]$ such that $z_{m}^{\prime}\left(x_{m}\right)=0$. Since the $z_{m}$ are bounded and $f_{m}$ converges uniformly to $f$, by standard theory for initial value problems (see [8, Lemma 3.1]) the $z_{m}$ have a subsequence convergent to a solution $z$ of (1.1) on some maximal subinterval $J$ of $[a, b]$. Either $J=[a, b]$ and $z$ is the required solution or $z^{\prime}$ is unbounded on $J$.

Suppose $z^{\prime}$ is unbounded on $J$. By the theory of initial value problems there is $c \in[a, b]$ and $\delta>0$ such that either $J \supset(c, c+\delta)$ and $\left|z^{\prime}\left(c^{+}\right)\right|=\infty$ or $J \supset(c-\delta, c)$ and $\left|z^{\prime}\left(c^{-}\right)\right|=\infty$. Consider the case $J \supset(c, c+\delta)$ and $z\left(c^{+}\right)=-\infty$. The proof is a variation on part of the proof of Theorem 3.12. Now $\alpha(x) \leq z(x) \leq \beta(x)$, for all $x \in(c, c+\delta)$ and $z\left(c^{+}\right)$exists. By the Mean Value Theorem $\mathscr{D} \mathscr{R} z(c)=-\infty$ and there is $x_{1} \in(c, c+\delta)$ such that $z^{\prime}\left(x_{1}\right)>\left(z\left(x_{1}\right)-z\left(c^{+}\right)\right) /\left(x_{1}-c\right)$. Let $w$ be the solution of (3.9) satisfying

$$
w\left(x_{1}\right)=z\left(x_{1}\right), \quad w^{\prime}\left(x_{1}\right)=\left(z\left(x_{1}\right)-z\left(c^{+}\right)\right) /\left(x_{1}-c\right) .
$$

As $\mathscr{D} \mathscr{R} z(c)=-\infty, z^{\prime}\left(x_{1}\right)>w^{\prime}\left(x_{1}\right)$ and $z$ is a solution on $(c, c+\delta)$, $0<\sup \left\{w(x)-z(x): c<x<x_{1}\right\}=w\left(x_{2}\right)-z\left(x_{2}\right)$ and $w^{\prime}\left(x_{2}\right)=z^{\prime}\left(x_{2}\right)$ for some $x_{2} \in\left(c, x_{1}\right)$. Thus $z\left(x_{2}\right) \leq w\left(x_{2}\right)$ so

$$
\begin{aligned}
w^{\prime \prime}\left(x_{2}\right) & =h\left(x_{2}, w\left(x_{2}\right), w^{\prime}\left(x_{2}\right)\right) \geq h\left(x_{2}, z\left(x_{2}\right), w^{\prime}\left(x_{2}\right)\right) \\
& >f\left(x_{2}, z\left(x_{2}\right), z^{\prime}\left(x_{2}\right)\right)=z^{\prime \prime}\left(x_{2}\right),
\end{aligned}
$$

since (3.10) holds for all $y$. This is a contradiction hence $z^{\prime}\left(c^{+}\right) \neq-\infty$.

Using (3.8), (3.11) and a similar variation on another part of the proof of Theorem 3.12, the case $z^{\prime}\left(c^{+}\right)=\infty$ leads to the inequalities (3.15), a contradiction. Thus $z^{\prime}\left(c^{+}\right) \neq \infty$.

The other cases $J \supset(c-\delta, c)$ and $\left|z^{\prime}\left(c^{-}\right)\right|=\infty$ follow similarly. Thus $z$ is the required solution.

Indeed, if (3.10) and (3.11) hold for all $y$, then (1.1) and (1.4) has a solution for all $A$ and $B$ as can be seen from the above arguments.

The following result is an easy consequence of Theorem 3.6.

THEOREM 3.14. Let $\alpha$ be a bounded subfunction for $y^{\prime \prime}=f\left(x, y, y^{\prime}\right)$ on $[a, b]$. Assume that for all $\left[x_{1}, x_{2}\right] \subset[a, b]$ and $y_{i} \geq \alpha\left(x_{i}\right), i=1,2$, the boundary value problem (1.1) and (1.2) has at least one solution, then $\alpha$ is continuous on $(a, b), \alpha^{\prime}$ exists in the reals except possibly for a countable number of points $x \in N$ at which $\infty>\mathscr{D} \mathscr{R} \alpha(x)>$ $\mathscr{D} \mathscr{L} \alpha(x)>-\infty$.

4. Examples. In the following example we construct a function $z$ on $[0,1]$ which is simultaneously a subfunction and a superfunction on 
$[0,1]$ for a function $f \in C_{\text {loc }}^{1,1}\left(\mathbf{R}^{3}\right)$ which we construct. The function $z$ has a denumerable number of discontinuities and satisfies $z^{\prime}(x)=\infty$, for all $x \in P$, the Cantor ternary set. This shows that Theorem 3.7 is sharp.

Example 4.1. Let $[a, b]=[0,1], P$ be the Cantor ternary set, and $[0,1] \backslash P=\bigcup_{n, j} I_{n, j}=I$, where $I_{n, j}=\left(a_{n, j}, b_{n, j}\right)$ are the components of $I$ of length $3^{-n}$. Set $x_{n, j}=\left(a_{n, j}+b_{n, j}\right) / 2$ and define $\Phi_{n, j}: \mathbf{R} \rightarrow \mathbf{R}$ by

$$
\Phi_{n, j}= \begin{cases}0, & \text { if } x \leq a_{n, j}, \\ n\left(x-a_{n, j}\right)+\left(1+\pi^{-1} \arcsin \left\{2\left(x-x_{n, j}\right) 3^{n}\right\}\right), & \text { if } a_{n, j}<x<b_{n, j}, \\ n 3^{-n}+2, & \text { if } x \geq b_{n, j} .\end{cases}
$$

Set $w(x)=\sum_{n, j}\left(\frac{2}{5}\right)^{-n} \Phi_{n, j}(x)$. Arguing as in [5, pp. 138-139] we see that $w$ is a monotonic, nondecreasing continuous function with $w^{\prime}(x)=\infty$, for all $x \in P$. Let

$$
H=\left\{(x, u, p) \in \mathbf{R}^{3}: x \in I, u=w(x),\left|p-w^{\prime}(x)\right| \leq 1\right\} .
$$

Since $w^{\prime}$ is continuous in $I$ and $\lim _{x \rightarrow c} w^{\prime}(x)=\infty$, for all $c \in P$ we see that $H$ is a closed set. Define $g: H \rightarrow \mathbf{R}$ by $g(x, w(x), p)=w^{\prime \prime}(x)$. Let $F=\left\{(x, u, p) \in \mathbf{R}^{3}:(x, u+v, p) \in H\right.$ some $\left.v \in \mathbf{R}\right\}$. Thus $F$ is a closed set. Extend $g$ to $F$ by setting $g(x, u, p)=w^{\prime \prime}(x)+u-w(x)$. Now let $g_{u}(x, u, p)=1, g_{p}(x, u, p)=0$ and $g_{x}(x, u, p)=w^{\prime \prime \prime}(x)-w^{\prime}(x)$. Then $g \in C_{\text {loc }}^{1,1}(F)$ so by the Whitney extension procedure (see [16, p. 176]) we may extend $g$ to $\mathbf{R}^{3}$ so that $g \in C_{\mathrm{loc}}^{1,1}\left(\mathbf{R}^{3}\right)$. We show that $w$ is a superfunction. The idea of the proof is similar to part of the proof of Theorem 3.10 and to the proof that $w$ is a subfunction, which we omit. Now $w$ satisfies the Assumption 3.4' obtained from Assumption 3.4 by reversing all inequalities and interchanging max and min. Let $\left[x_{1}, x_{2}\right] \subset[0,1]$ and let $y \in C^{2}\left[x_{1}, x_{2}\right]$ be a solution of $y^{\prime \prime}=g\left(x, y, y^{\prime}\right)$ on $\left[x_{1}, x_{2}\right]$ with $y\left(x_{i}\right) \leq w\left(x_{i}\right)$ for $i=1,2$ and $y\left(x_{0}\right)>w\left(x_{0}\right)$ some $x_{0} \in\left(x_{1}, x_{2}\right)$. From the analogue of Lemma 3.5 for superfunctions we see that

$$
0<l=\sup \left\{y(x)-w(x): x_{1} \leq x \leq x_{2}\right\}=y\left(x_{4}\right)-w\left(x_{4}\right)
$$

some $x_{4} \in\left(x_{1}, x_{2}\right)$ and $y^{\prime}\left(x_{4}\right)=w^{\prime}\left(x_{4}\right)$. As $w^{\prime}\left(x_{4}\right)$ exists $x_{4} \in I$ and $y^{\prime \prime}\left(x_{4}\right)-w^{\prime \prime}\left(x_{4}\right) \leq 0$. Now

$$
\begin{aligned}
y^{\prime \prime}\left(x_{4}\right) & =g\left(x_{4}, y\left(x_{4}\right), y^{\prime}\left(x_{4}\right)\right) \\
& >g\left(x_{4}, w\left(x_{4}\right), w^{\prime}\left(x_{4}\right)\right)=w^{\prime \prime}\left(x_{4}\right),
\end{aligned}
$$


since $g$ is strictly increasing with respect to $u$ on $F, x^{\prime}\left(x_{4}\right)=w^{\prime}\left(x_{4}\right)$ and $y\left(x_{4}\right)>w\left(x_{4}\right)$. This is a contradiction so that $w$ is a superfunction.

Thus we have constructed $g \in C_{\mathrm{loc}}^{1,1}\left([0,1] \times \mathbf{R}^{3}\right)$ and a continuous function $w$ with $w^{\prime}(x)=\infty$ on $P$ which is simultaneously a subfunction and a superfunction for $g$. To construct $f$ and the function $z$ proceed as follows. Let $x_{m}=1-3^{-m}$ for $m=1,2, \ldots$ and let $\Psi: \mathbf{R} \rightarrow \mathbf{R}$ be defined by

$$
\Psi(x)= \begin{cases}\sum_{m ; x_{m}<x} 3^{-2 m}, & \text { for } x>\frac{2}{3}, \\ 0, & \text { for } x \leq \frac{2}{3}\end{cases}
$$

and let $\Phi:[0,1] \rightarrow \mathbf{R}$ be defined by $\Phi(x)=\left(\Psi\left(x^{+}\right)+\Psi\left(x^{-}\right)\right) / 2$. It is easy to check that $\Phi$ is nondecreasing with discontinuities at $x_{m}$, for all $m=1,2, \ldots$, that $\Phi^{\prime}(x)=0$, for all $x \in[0,1) \backslash \bigcup_{m=1}^{\infty}\left\{x_{m}\right\}$, that $\mathscr{D} \mathscr{R} \Phi(x)$ exists for all $x \in[0,1)$, and that $\mathscr{D} \mathscr{L} \Phi(x)$ exists for all $x \in(0,1]$; indeed, $\mathscr{D} \mathscr{R} \Phi(1)=0$. Set $z(x)=w(x)+\Phi(x)$ and $f(x, u, p)=g(x, u-\Phi(x), p)$ for all $(x, u, p) \in F$. Arguing as before we may extend $f$ to $\mathbf{R}^{3}$ such that $f \in C_{\text {loc }}^{1,1}\left(\mathbf{R}^{3}\right)$ and may show that $z$ has the required properties.

Now we construct $f \in C_{\text {loc }}^{0,1}\left(\mathbf{R}^{3}\right)$ and a function $z_{1}$ on $[0,1]$ which is given by(1.7) but is not a superfunction for $f$ on $[0,1]$. The function $z_{1}$ has a denumerable number of discontinuities and $z_{1}^{\prime}(x)=\infty$, for all $x \in P$, the Cantor ternary set. As $f$ has the local unique boundary value property Theorem 3.9 applies. This shows that Theorem 3.9 is sharp.

EXAMPLE 4.2. Let $z$ be given by Example 4.1. Let $y_{1}(x)=z(x)$ and $y_{2}(x)=z(x)+1$ on $[0,1]$. Let $h(x)=243(x-4 / 9)(5 / 9-x)$, $v_{1}(x)=y_{1}(x)+h(x)$ and $v_{2}(x)=y_{2}(x)-h(x)$ on $[4 / 9,5 / 9]=J$. Thus $y_{1}(1 / 2)<v_{2}(1 / 2)<v_{1}(1 / 2)<v_{2}(1 / 2), v_{1}\left(\frac{3+j}{9}\right)=y_{1}\left(\frac{3+j}{9}\right)$ and $y_{1}(x) \leq v_{i}(x) \leq y_{2}(x)$ on $J$, for $i, j=1,2$. Let

$$
G_{i}=\left\{(x, u, p): x \in J, u=v_{i}(x), p=v_{i}^{\prime}(x)\right\} \text { for } i=1,2 .
$$

Let $K=I \cap\{[0,7 / 18] \cup[11 / 18,1]\}$ and

$$
G_{3}=\left\{(x, u, p): x \in K, p=y_{1}^{\prime}(x)=y_{2}^{\prime}(x), y_{1}(x) \leq u \leq y_{2}(x)\right\} .
$$

Let

$$
G_{4}=\left\{(x, u, p): x \in I, p=y_{1}^{\prime}(x)=y_{2}^{\prime}(x), y_{2}(x) \leq u\right\}
$$

and

$$
G_{5}=\left\{(x, u, p): x \in I, p=y_{1}^{\prime}(x)=y_{2}^{\prime}(x), u \leq y_{1}(x)\right\}
$$


Thus $G_{i}$ is a closed set for $1 \leq i \leq 5$. Define $f$ on $G_{1} \cup G_{2}$ by $f\left(x, v_{i}(x), v_{i}^{\prime}(x)\right)=v_{i}^{\prime \prime}(x)$, for $i=1,2$. Define $f$ on $G_{3} \cup G_{4} \cup G_{5}$ by

$$
f(x, u, p)=y_{1}^{\prime \prime}(x)+ \begin{cases}u-y_{1}(x), & \text { if }(x, u, p) \in G_{5}, \\ 0, & \text { if }(x, u, p) \in G_{3}, \\ u-y_{2}(x), & \text { if }(x, u, p)=G_{4} .\end{cases}
$$

Thus $f$ is locally lipschitz on $\bigcup_{i=1}^{5} G_{i}$ and $f$ can be extended by the Whitney procedure to a locally lipschitz function on $\mathbf{R}^{3}$. The proof that $y_{2}$ is a superfunction for $f$ is similar to that in Example 4.1. A similar argument shows $y_{1}$ is a subfunction for $f$. Set

$$
\begin{aligned}
z_{1}(x)=\sup \left\{\Phi(x): y_{1}(x) \leq\right. & \Phi(x) \leq y_{2}(x) \\
& \text { and } \Phi \text { a subfunction for } f \text { on }[0,1]\} .
\end{aligned}
$$

Since $f \in C_{\text {loc }}^{0,1}\left(\mathbf{R}^{3}\right)$ it has the local unique boundary value property so Theorem 3.9 applies. To see that $z_{1}$ is not a superfunction we observe that since $z_{1}$ is a subfunction and $v_{2}$ is a solution on $[4 / 9,5 / 9]$ with $v_{2}(4 / 9)=y_{2}(4 / 9) \geq z_{1}(4 / 9)$ and $v_{2}(5 / 9)=y_{2}(5 / 9) \geq z_{1}(5 / 9)$ then $z_{1}(1 / 2) \leq v_{2}(1 / 2)$. If $z_{1}$ is a superfunction a similar argument shows that $z_{1}(1 / 2) \geq v_{1}(1 / 2)$, but $v_{2}(1 / 2)<v_{1}(1 / 2)$, a contradiction, so $z_{1}$ is not a superfunction. To see that $z_{1}$ has the required smoothness properties we show that $z_{1}(x)=1+z(x)$, for all $x \in(2 / 3,1]$. To prove this we show that

$$
\Phi(x)= \begin{cases}y_{2}(x), & \text { if } x \in(2 / 3,1] \\ y_{1}(x), & \text { if } x \in[0,2 / 3]\end{cases}
$$

is a subfunction. Suppose $\Phi$ is not a subfunction. Let $\left[x_{1}, x_{2}\right] \subset$ $[0,1]$ and $y$ be a solution of $y^{\prime \prime}=f\left(x, y, y^{\prime}\right), y\left(x_{i}\right) \geq \Phi\left(x_{i}\right)$, for $i=$ $1,2, y\left(x_{0}\right)<\Phi\left(x_{0}\right)$ some $x_{0} \in\left(x_{1}, x_{2}\right)$. Since $\Phi$ satisfies the conditions of Assumption 3.4, Lemma 3.5 shows that

$$
0<l=\sup \left\{\Phi(x)-y(x): x_{1} \leq x \leq x_{2}\right\}=\Phi\left(x_{4}\right)-y\left(x_{4}\right)
$$

some $x_{4} \in\left(x_{1}, x_{2}\right)$ and $\Phi^{\prime}\left(x_{4}\right)=y^{\prime}\left(x_{4}\right)$. Now since $\Phi^{\prime}\left(x_{4}\right)$ exists $x_{4} \in I$. Now $y\left(x_{4}\right)<z\left(x_{4}\right)$ is impossible since $z$ is a subfunction on $[0,1]$. Thus $x_{4} \in I_{n, j} \subset(2 / 3,1]$, some $n, j, z\left(x_{4}\right) \leq y\left(x_{4}\right)<1+z\left(x_{4}\right)=\Phi\left(x_{4}\right)$ and $y^{\prime}\left(x_{4}\right)=z^{\prime}\left(x_{4}\right)$. Since $f \in C_{\mathrm{loc}}^{0,1}\left(\mathbf{R}^{3}\right)$ the initial value problem $y^{\prime \prime}=f\left(x, y, y^{\prime}\right), y^{\prime}\left(x_{4}\right)=z^{\prime}\left(x_{4}\right), y\left(x_{4}\right)$ fixed, has a unique solution and this is $z(x)+y\left(x_{4}\right)-z\left(x_{4}\right)$ on $I_{n, j}$, by the construction of $f$. Thus $I_{n, j} \subset\left[x_{1}, x_{2}\right]$ but $y \in C^{2}\left[x_{1}, x_{2}\right]$, a contradiction. Thus $\Phi$ is a subfunction and the result follows. 
Now we construct a function $f_{2} \in C_{\mathrm{loc}}^{0,1}\left([0,1] \times \mathbf{R}^{2}\right)$ and a function $\alpha$ on $[0,1]$ which is a subfunction for $f_{2}$ on $[0,1]$. The function $\alpha$ has a denumerable number of discontinuities, $\alpha^{\prime}(x)=\infty$, for all $x \in P$, the Cantor ternary set and $\mathscr{D} \mathscr{R} \alpha(x)>\mathscr{D} \mathscr{L} \alpha(x)$ at a dense set of points. This shows that Theorem 3.6 is sharp.

EXAMPLE 4.3. Let $I, P, f$, and $z$ be as given in Example 4.1. Let $\left\{q_{m} \in I: m=1,2, \ldots\right\}$ be the rationals in $I$. Let $\Psi: \mathbf{R} \rightarrow \mathbf{R}$ be given by $\Psi(x)=\sum_{m ; q_{m} \leq x} 2^{-m}\left(x-q_{m}\right)$. It is easy to see that $|\Psi(x)-\Psi(t)| \leq$ $|x-t|$ for all $x, t \in \mathbf{R}$, that $\mathscr{D} \mathscr{R} \Psi\left(q_{m}\right)-\mathscr{D} \mathscr{L} \Psi\left(q_{m}\right)=2^{-m}>0$ and $\Psi^{\prime}(x)$ exists for all $x \notin\left\{q_{m}: m=1,2, \ldots\right\}$. As $\mathscr{D} \mathscr{R} \Psi(x)$ is nondecreasing $D_{+} \mathscr{D} \mathscr{R} \Psi(x) \geq 0$ for all $x \in \mathbf{R}$. Set $\alpha(x)=z(x)+\Psi(x)$ on $[0,1]$ so $|\mathscr{D} \mathscr{R} \alpha(x)-\mathscr{D} \mathscr{R} z(x)| \leq 1$, for all $x \in I$. Define $f_{2}:[0,1] \times \mathbf{R}^{2} \rightarrow \mathbf{R}$ by $f_{2}(x, u, p)=f(x, u-\Psi(x), p)$. Since $f \in C_{\text {loc }}^{1,1}\left(\mathbf{R}^{3}\right)$ and $\psi$ is lipschitz it follows that $f_{2} \in C_{\text {loc }}^{0,1}\left([0,1] \times \mathbf{R}^{2}\right)$. Also

$$
\begin{aligned}
D_{+} \mathscr{D} \mathscr{R} \alpha(x) & \geq D_{+} \mathscr{D} \mathscr{R} z(x) \geq f(x, z,(x), \mathscr{D} \mathscr{R} z(x)) \\
& =f(x, \alpha(x)-\Psi(x), \mathscr{D} \mathscr{R} \alpha(x)) \\
& =f_{2}(x, \alpha(x), \mathscr{D} \mathscr{R} \alpha(x)), \text { for all } x \in I .
\end{aligned}
$$

It follows from the proof of Theorem 3.10 that $\alpha$ is a subfunction for $f_{2}$ on $[0,1]$. Clearly $\alpha$ has the required properties.

Acknowledgment. The author would like to thank Michael Meier for helpful comments, especially for drawing his attention to errors in the draft version of Theorem 3.12.

\section{REFERENCES}

[1] K. Ako, Subfunctions for ordinary differential equations, J. Fac. Sci. Univ. Tokyo, I, 9 (1965), 17-43.

[2] Subfunctions for ordinary differential equations II, Funkcialaj Ekvacioj, 10 (1967), 145-162.

[3] P. B. Bailey, L. F. Shampine and P. E. Waltman, Nonlinear Two Point Boundary Value Problems, Academic Press, New York, 1968.

[4] S. R. Bernfeld and V. Lakshmikantham, An Introduction to Nonlinear Boundary Value Problems, Academic Press, New York, 1974.

[5] R. P. Boas, A Primer of Real Functions, in Carus Mathematical Monographs. The Mathematical Association of America, New York, 1961.

[6] F. F. Bonsall, The characterization of generalized convex functions, Quart. J. Math. Oxford Ser. (2), 1 (1950), 100-111.

[7] J. W. Green, Generalized convex functions, Proc. Amer. Math. Soc., 4 (1953), 391-396.

[8] J. K. Hale, Ordinary Differential Equations, Wiley-Interscience, New York, 1969. 
[9] L. K. Jackson, Subfunctions and second-order ordinary differential inequalities, Advances in Math., 2 (1968), 307-363.

[10] G. Klassen, Dependence of solutions on boundary conditions for second order ordinary differential equations, J. Differential Equations, 7 (1970), 24-33.

[11] O. Perron, Ein Neuer Existenzbeweis fur die Integrale der Differentialgleichung $y^{\prime}=f(x, y)$, Math. Ann., 76 (1915), 471-484.

[12] Eine neue Behandlung der ersten Randwert-aufgabe fur $\Delta u=0$, Math. Z., 18 (1923), 42-54.

[13] M. M. Piexoto, Generalized convex functions and second order differential inequalities, Bull. Amer. Math. Soc., 55 (1949), 563-572.

[14] W. T. Reid, Variational aspects of generalized convex functions, Pacific J. Math., 9 (1959), 571-581.

[15] F. Riesz and B. Sz-Nagy, Functional Analysis, Ungar, New York, 1955.

[16] E. M. Stein, Singular Integrals and Differentiability Properties of Functions, Princeton University Press, New Jersey, 1970.

Received August 28, 1987.

UNIVERSITY OF QUEENSLAND

St. Lucia, 4067, QueEnsLand

Australia 



\section{PACIFIC JOURNAL OF MATHEMATICS EDITORS}

\author{
V. S. VARADARAJAN \\ (Managing Editor) \\ University of California \\ Los Angeles, CA 90024-1555-05 \\ Herbert Clemens \\ University of Utah \\ Salt Lake City, UT 84112 \\ THOMAS ENRIGHT \\ University of California, San Diego \\ La Jolla, CA 92093
}

R. FINN

Stanford University

Stanford, CA 94305

HeRMANN FlaschKa

University of Arizona

Tucson, AZ 85721

VAUGHaN F. R. Jones

University of California

Berkeley, CA 94720

STEVEN KeRCKHOFF

Stanford University

Stanford, CA 94305

\author{
ROBION KIRBY \\ University of California \\ Berkeley, CA 94720 \\ C. C. MOORE \\ University of California \\ Berkeley, CA 94720 \\ HAROLD STARK \\ University of California, San Diego \\ La Jolla, CA 92093
}

\section{ASSOCIATE EDITORS}
R. ARENS
E. F. BECKENBACH
B. H. NEUMANN
F. WOLF
K. YoshidA (1906-1982)

\section{SUPPORTING INSTITUTIONS}

UNIVERSITY OF ARIZONA

UNIVERSITY OF BRITISH COLUMBIA

CALIFORNIA INSTITUTE OF TECHNOLOGY

UNIVERSITY OF CALIFORNIA

MONTANA STATE UNIVERSITY

UNIVERSITY OF NEVADA, RENO

NEW MEXICO STATE UNIVERSITY

OREGON STATE UNIVERSITY
UNIVERSITY OF OREGON

UNIVERSITY OF SOUTHERN CALIFORNIA

STANFORD UNIVERSITY

UNIVERSITY OF HAWAII

UNIVERSITY OF TOKYO

UNIVERSITY OF UTAH

WASHINGTON STATE UNIVERSITY

UNIVERSITY OF WASHINGTON 


\section{Pacific Journal of Mathematics}

Vol. 140, No. $1 \quad$ September, 1989

Michel Brestovski, Algebraic independence of solutions of differential

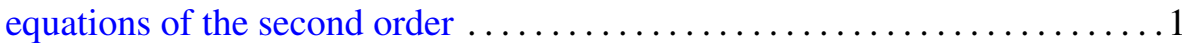

Bohumil Cenkl, Cohomology operations from higher products in the de

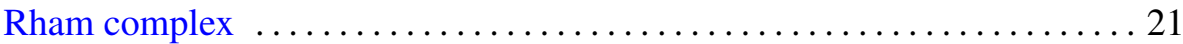

Gustavo Corach and Daniel Suarez, Generalized rational convexity in

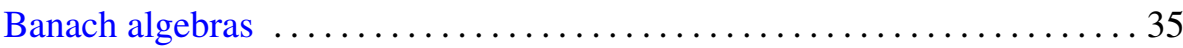

Keresztély Corrádi and Sándor Szabó, A new proof of Rédei’s theorem . . . 53

Steven R. Costenoble and Stefan Waner, Equivariant orientations and

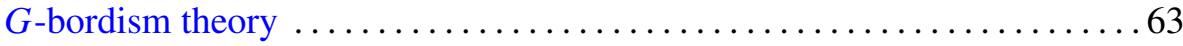

Angel Granja, Apéry basis and polar invariants of plane curve

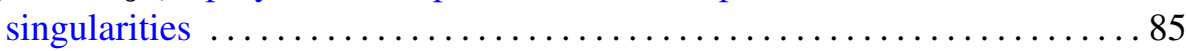

Young Soo Jo, Isometries of tridiagonal algebras .................. 97

Ronald Leslie Lipsman, Harmonic analysis on exponential solvable homogeneous spaces: the algebraic or symmetric cases $\ldots \ldots \ldots \ldots \ldots 117$

Erich Miersemann, On the behaviour of capillaries at a corner 149

Marian Nowak, On the finest Lebesgue topology on the space of essentially

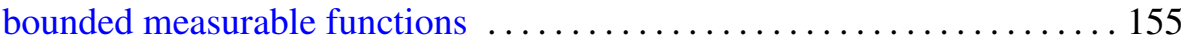

Pascal J. Thomas, Hardy interpolating sequences of hyperplanes ........ 163

H. Bevan Thompson, Differentiability properties of subfunctions for second order ordinary differential equations 